\title{
Spatial orientation assessment in preschool children: Egocentric and allocentric frameworks
}

\author{
Q1 C. Fernandez-Baizan ${ }^{a, b}$, J. L. Arias ${ }^{a, b}$, and M. Mendez ${ }^{a, b}$
} ${ }^{a}$ Department of Psychology, University of Oviedo, Oviedo, Spain; ${ }^{b}$ Instituto de Neurociencias del Principado de Asturias (INEUROPA),
Oviedo, Spain

\begin{abstract}
Spatial orientation is an important function in daily life because it allows us to reach a target place when moving through our environment, using self-centered (egocentric) or environmental information (allocentric). Compared to other cognitive functions, spatial orientation has been studied less in preschool ages. Some brain areas, such as the hippocampus and the temporal as well as the parietal and frontal cortices, are involved in spatial orientation. Therefore, when these brain regions are altered in neurological conditions or in atypical development in children, we would expect impairment of spatial abilities. The aim of this study is to review studies, published in recent years, that use egocentric and allocentric spatial orientation tasks for assessing spatial memory in preschool children, with the final goal of finding out which tests could be included in a clinical neuropsychological evaluation. We observed that although egocentric spatial orientation emerges first during development, allocentric spatial orientation tasks are employed at very early ages. Most of these tasks are performed in real environments, allowing children's self-movements and using environmental modifications, but technologies such as virtual or augmented reality are increasingly used. Other aspects are discussed, such as the lack of consensus in the nomenclature, the difficulty of tracing the course of development of spatial orientation, or the ecological validity of the tests used. We finally observed that there is greater interest in studying the allocentric framework than the egocentric one, which makes it difficult to compare the use of the two frames of reference during a neuropsychological evaluation in preschool-aged children.
\end{abstract}

\section{KEYWORDS}

Frames of reference;

preschoolers; spatial

memory; spatial orientation

topographical disorienta-

tion; young children

\section{Introduction}

In our daily lives, we need to employ several cognitive functions that work with visual and spatial information, ranging from simple tasks, such as remembering in which drawer of the room we put an object, to complex tasks, such as remembering the path we need to follow to reach a distant place. The former example describes memory for locations, related to object recognition, recall of positions of objects, or knowing when an object has been moved from its original position (Bocchi et al., 2018). The latter example refers to spatial orientation or spatial navigation, which is the aim of the present review. Spatial orientation allows us to find a target place by moving through the environment to reach the target location. To do this, it is necessary to remember the location, use information about the direction, angle, or distance, and specify the location with regard to two cues: a stable and unchanging reference point and/or a subject who moves (Vasilyeva \& Lourenco, 2012; Waismeyer \& Jacobs, 2013). Thus, spatial orientation not only requires coding such information or key points in the environment, but also knowing how to use them to relocate to a previously known or visited place. Hence, all of this information allows us to eventually reach our target location, identifying our surrounding environment and our location and reorienting in previously known places. Therefore, we can see that spatial orientation involves location memory, and this function is more complex than visual orientation.

\section{Egocentric vs allocentric spatial orientation}

For spatial navigation, we mainly employ two frameworks: egocentric and allocentric. The egocentric system involves taking our own body as the reference 
center, so that all locations are related to our own point of view, whereas the allocentric system employs elements in the environment regardless of our own position (Burgess, 2008; O'Keefe, Nadel, \& Lynn, 1978; Vasilyeva \& Lourenco, 2012). A third frame of reference has been pointed out, the heterocentric framework, which takes the perspective of a third person (Ruby \& Decety, 2001). However, this framework has not been considered in the present review. The egocentric framework is related to a concept known as dead reckoning, or path integration, the ability to update and track our own movements by codifying our speed or acceleration, as well as distances, turns, and directions, called idiothetic signals (Montello, 2005). This capacity requires awareness of our initial location, and it involves vestibular, kinesthetic, and optic systems (Newcombe, Huttenlocher, Drummey, \& Wiley, 1998; van den Brink \& Janzen, 2013). The allocentric framework is related to the ability to recall and recognize landmarks-allothetic signals-to finally be able to make mental maps as representations of the surrounding environment (Burgess, Maguire, \& O'Keefe, 2002; Montello, 2005; O'Keefe et al., 1978). These external allothetic cues can be coincident or not coincident: coincident cues code the location's properties or the reference landmarks that coincide with the target place, whereas noncoincident cues refer to relative positions that require the ability to employ distances and directions related to the target place (Waismeyer \& Jacobs, 2013). Moreover, when we talk about a noncoincident landmark, we can refer to environmental landmarks inside the task enclosure or near a target location, called proximal cues or intramaze landmarks, or we can refer to external environmental cues not located in the enclosure or far from the target location, called distal cues or boundary landmarks. Therefore, if we hide a toy under a box decorated with a distinctive pattern, children could memorize the position of the box on the floor of the room and its distinctive feature (a coincident cue), but they could also code that the target box is next to the teddy bear (a proximal noncoincident cue) or just below the window (a distal noncoincident cue).

For completely functional spatial navigation, it is not enough to have these environmental cues and our own organism's cues, statically or in locomotion. Instead, it is necessary to integrate and combine them to eventually recreate integral images of spatial representations (Nardini, Jones, Bedford, \& Braddick, 2008). Thus, taking into account that the egocentric framework is the first to emerge in development (Acredolo, 1978; Acredolo \& Evans, 1980; Piaget \&
Inhelder, 1967), and based on the model of environmental knowledge acquisition (Siegel \& White, 1975), the development of the allocentric framework and its association with the egocentric framework takes place in the following way. First, in landmark knowledge acquisition, we can recognize landmarks in the environment, but not to employ directional information about them, such as their location and their association with other stimuli and the environment. Second, in route knowledge, we can employ egocentric information in combination with consecutive landmarks, allowing us to follow a route. Third, in survey knowledge, we can memorize landmarks in an organized mental map. Therefore, the allocentric response starts with remembering landmarks, then combining them with egocentric information, and concluding with the creation of mental maps, indicating that allocentric orientation is fully developed, which occurs at about the age of seven (Overman, Pate, Moore, \& Peuster, 1996). Therefore, it seems relevant to assess spatial orientation development before the age of seven.

\section{Typical tasks to measure egocentric and allocentric spatial orientation}

Prototypical tasks used for the evaluation of the egocentric and allocentric frames of reference take place in rooms specifically equipped for the test. In the case of the egocentric framework, the room or enclosure has not environmental cues in order to force the use of turns and body movements to guide behavior. In the case of the allocentric framework, visual cues would be added. The final aim of allocentric tasks is usually for the child to find a stimulus or toy in a hidden place within the room, guided either by his own turns and/or body movements in the egocentric framework, or by the cues located in the environment in the allocentric framework.

\section{Development of spatial orientation related functions}

Regarding brain development during childhood and spatial orientation improvements, other early sensorial and cognitive functions have to develop first. That is, to correctly perceive the environment, discriminate landmarks, locate objects spatially, etc., children need to move their eyes and head across their visual field in order to explore it. On eye-tracking tasks, infants are encouraged to follow a stimulus across their visual field. This requires infants to able to voluntarily control their eye movements. Newborns are only able to 
perform eye tracking tasks, fixing their attention on a specific stimulus, although this ability improves significantly at 3 months (Clohessy, Posner, \& Rothbart, 2001 ) and continues to improve until 6 months (Reynolds \& Romano, 2016). Therefore, between 3 and 6 months of age, infants begin to have the ability to voluntarily control their eye movements, which is related to exploring their visual field. At 6 months, infants are able to maintain their attentional focus for longer periods of time (Reynolds \& Romano, 2016), and at the age of one year, they start to inhibit nonrelevant stimulation (Garon, Bryson, \& Smith, 2008). From this age onward, infants can sustain their attentional focus longer and tolerate more interfering information (Garon et al., 2008). At 6 months old, infants also start to detect and anticipate changes in the positions of objects previously shown, as a very first sign of visuospatial memory (Gilmore \& Johnson, 1995; Oakes, Hurley, Ross-Sheehy, \& Luck, 2011; Reznick, Morrow, Goldman, \& Snyder, 2004). This function continues improving significantly between 6 and 8 months (Pelphrey et al., 2004), but infants still have a high rate of mistakes when trying to locate an object between two possible positions until 9 months old (Diamond \& Goldman-Rakic, 1989). From 9 months to one year, searching errors almost disappear (Diamond \& Goldman-Rakic, 1989). In addition, infants start to be able to tolerate a longer delay period, a period of time where the child is not allowed to initiate searching behavior, from 2 seconds at 6 months to 10 seconds at 12 months (Diamond \& Goldman-Rakic, 1989; Pelphrey et al., 2004; Reznick et al., 2004).

Some brain areas participate in the development of spatial orientation. Hippocampal maturity has been associated with allocentric memory enhancement, due to projections from the entorhinal cortex to the CA1 area (Lambert, Lavenex, \& Banta Lavenex, 2015), which occur at 2 years of age, approximately.

\section{Spatial orientation and brain structure and function}

In adult humans, on the one hand, the allocentric orientation has mainly been related to the hippocampus, parahippocampus, retrospenial, and inferior and superior temporal cortex, but also to the frontal, parietal, and occipital cortex (Boccia, Nemmi, \& Guariglia, 2014; Chen et al., 2014; Committeri et al., 2004; Doeller, King, \& Burgess, 2008; Nemmi, Boccia, \& Guariglia, 2017; Saj et al., 2014; Zaehle et al., 2007). On the other hand, the egocentric orientation is associated with the precuneus, inferior, and superior parietal cortex, superior temporal cortex, and frontal cortex, as well as some subcortical structures, such as the striatum, thalamus, and insula (Chen et al., 2014; Doeller et al., 2008; Moulinet et al., 2016; Neggers, Van der Lubbe, Ramsey, \& Postma, 2006; Nemmi et al., 2017; Zaehle et al., 2007). However, most of these areas are shared by both frameworks, especially the precuneus, parietal, temporal, and medial and superior frontal cortex (Chen et al., 2014; Neggers et al., 2006; Saj et al., 2014; Zaehle et al., 2007). Both strategies seem to involve a parieto-frontal bilateral network that mainly includes parietal and premotor regions, but can also include visual occipital regions (Gramann et al., 2010; Zaehle et al., 2007).

\section{Spatial orientation in pediatric neurological populations}

This aforementioned course of development of spatial abilities occurs in children with regular brain development. However, other cognitive abilities with a spatial component, such as visuospatial memory or visuospatial abilities, have been investigated more in children with early brain damage, atypical development, or other neurological conditions. For example, some difficulties have been found in these functions in premature children, compared to term-born children in (Beauchamp et al., 2008; Caravale, Tozzi, Albino, \& Vicari, 2005; Mürner-Lavanchy et al., 2014), due to a reduction in hippocampal volume (Aanes, Bjuland, Skranes, \& Løhaugen, 2015; Beauchamp et al., 2008). As mentioned previously, spatial orientation is a complex function based on the correct functioning of other sensory and cognitive systems, which leads us to imagine that space navigation may be affected in preterm children. For example, spatial orientation deficits have been observed in preterm, school-aged populations (Cimadevilla, Roldán, París, Arnedo, \& Roldán, 2014). In other clinical populations, such as Williams syndrome (Bernardino, Mouga, Castelo-Branco, \& Van Asselen, 2013; Broadbent, Farran, \& Tolmie, 2014; Nunes et al., 2013; Vicari, Bellucci, \& Carlesimo, 2003), Down syndrome (Lavenex et al., 2015), cerebral palsy (Belmonti, Fiori, Guzzetta, Cioni, \& Berthoz, 2015), and fetal alcohol syndrome (Hamilton, Kodituwakku, Sutherland, \& Savage, 2003), spatial orientation and visuospatial difficulties have been found as well. It is noteworthy that there is a disorder directly related to difficulties in spatial orientation development, that is, developmental topographical disorientation (DTD). Many cases of 
children and adults with this pathology have already been recorded (Bianchini et al., 2014; Conson et al., 2018; Iaria \& Barton, 2010; Iaria, Bogod, Fox, \& Barton, 2009; Iaria et al., 2005; Nemmi et al., 2015; Palermo, Foti, Ferlazzo, Guariglia, \& Petrosini, 2014; Palermo, Piccardi, et al., 2014), although we find that it has not yet been studied in depth compared to other neurodevelopmental disorders, and most of this population is detected in adulthood, pointing to a difficulty in detecting these problems in childhood at the present time. This pathology is characterized by problems related to topographic knowledge and environmental navigation, which may include difficulties such as recognizing landmarks, being aware of the position of the head, recognizing right-left directions, adapting to changes or alterations in previously learned routes, and, mainly, creating and later using cognitive maps (Iaria \& Barton, 2010). All these difficulties have been reported without presenting any type of cerebral lesion and with other neuropsychological functions intact.

Not only in clinical populations, but also in children with typical development, we find that spatial capacities play a vital role, due to their influence on academic achievement (Bull, Espy, \& Wiebe, 2008; Demir, Prado, \& Booth, 2014). Therefore, all these disorders or difficulties show the importance of using spatial orientation tests for preschool ages that allow us to detect early problems in this ability that could influence later cognitive development, academic aims, or even quality of life.

\section{Present review}

In spite of the vital importance of this capacity in our daily activities and in some neurological-related disorders, we find that, compared to other cognitive functions, not much attention has been paid to egocentric and allocentric evaluations during childhood. For all these reasons, it seems necessary to employ tests for these spatial orientation frames of reference from the earliest ages and throughout childhood, according to their brain development. Therefore, the aim of the present review is to identify and summarize the current state of knowledge on the egocentric and allocentric spatial orientation assessment tests for populations up to 6 years old that have been published in recent years. This review also aims to examine the methodological differences in spatial orientation assessment. Some tasks allow children to move, as we currently know that self-movement is relevant for orientation, while others do not allow movement or displacement. Similarly, some of the tasks use natural environments with some modifications, while others use technologies, such as computerized tasks, augmented reality or virtual reality. This review also sought to include a summary of the course of spatial orientation development, in order to clarify how this function improves in children with typical development. The knowledge about typical development could help to detect when there are dysfunctions, allowing clinical neuropsychologists to compare normal development with potential problems in spatial egocentric and allocentric function. Finally, this review also includes a critical discussion of the current panorama on the evaluation of spatial orientation in children, as well as limitations and directions for future research.

\section{Method}

A systematic literature review was conducted by searching in three psychological databases (Pubmed, PsycINFO, and Scholar Google) in order to identify peer-reviewed articles that employed spatial orientation tasks in children (age: 0-6 years). The studies included were published between 2000 and 2019. A description of the selection method is included in the supplementary material (see Figure 1).

\section{Results}

Sample description and study characteristics for the 49 studies are located in Table 1.

\section{Egocentric versus allocentric spatial orientation assessment}

Eighteen studies from 49 articles examined assessed egocentric spatial orientation using spatial tasks, out of (Bremner, Hatton, Foster, \& Mason, 2011; Bullens, Iglói, Berthoz, Postma, \& Rondi-Reig, 2010; Crowther, Lew, \& Whitaker, 2000; Gouteux, Vauclair, \& ThinusBlanc, 2001; Juan, Mendez-Lopez, Perez-Hernandez, \& Albiol-Perez, 2014; Kaufman \& Needham, 2011; Leplow et al., 2003; Lew, Bremner, \& Lefkovitch, 2000; Lew, Foster, \& Bremner, 2006; Nardini, Burgess, Breckenridge, \& Atkinson, 2006; Nardini, Jones, et al., 2008; Piccardi et al., 2014; Piccardi, Leonzi, D’Amico, Marano, \& Guariglia, 2014; Piccardi, Palermo, Bocchi, \& Guariglia, 2015; Ruggiero, D’Errico, \& Iachini, 2016; Vasilyeva \& Bowers, 2006; Waismeyer \& Jacobs, 2013; Yang, Merrill, \& Wang, 2019). In 7 of these articles the emission of a self-centered response is 


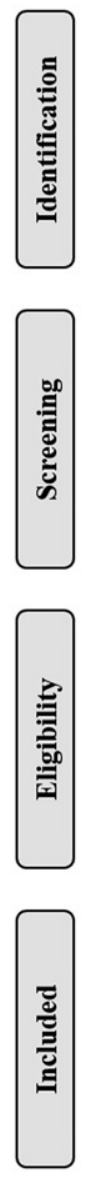

Additional records identified through other sources $(\mathrm{n}=0)$ $(\mathrm{n}=1860)$

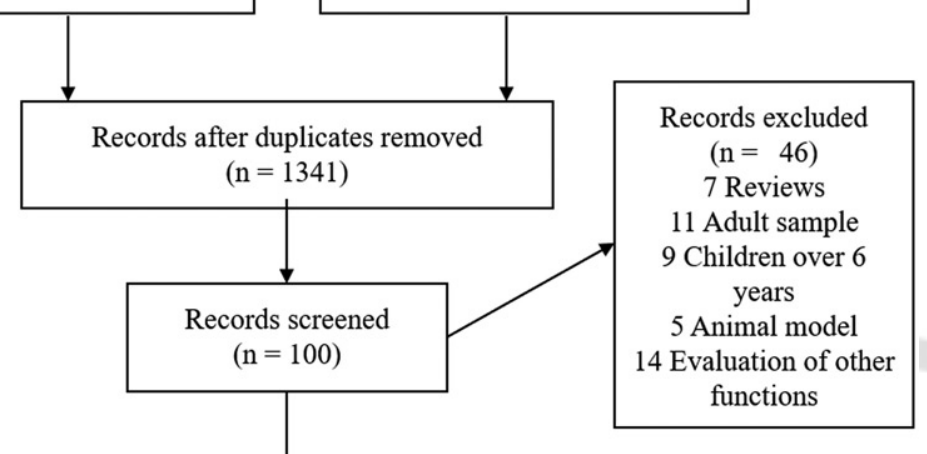

Studies included in qualitative synthesis $(n=49)$

Figure 1. Flow of full-text articles selected for revision according to the Preferred Reporting Items for Systematic Reviews and Meta-Analyses (PRISMA) guidelines.

considered erroneous or noneffective (Gouteux et al., 2001; Kaufman \& Needham, 2011; Leplow et al., 2003; Lew et al., 2006; Nardini, Jones, et al., 2008; Vasilyeva \& Bowers, 2006; Waismeyer \& Jacobs, 2013), because the main aim of these tasks is to know whether the children are capable of using external environmental landmarks. The only studies that used tasks that can only be solved using egocentric cues did not aim to analyze, as the main objective, the performance on this framework, but rather to measure the visuospatial span (Juan et al., 2014; Piccardi, Leonzi, et al., 2014; Piccardi, Palermo, et al., 2014), locating one or more objects among several possible locations, as well as walking according to a specific pattern through squares placed on the floor. The studies also assessed what type of egocentric information is used more effectively (Bremner et al., 2011) when visual flow or vestibular information, or egocentric information is available along with allocentric clues, hindering the use of egocentric strategy by itself (Yang et al., 2019).

Forty studies from 49 articles examined evaluated the allocentric spatial orientation. In 32 of these articles noncoincident landmarks were used (Bullens,
Iglói, et al., 2010; Bullens, Nardini et al., 2010; Bullens, Klugkist, \& Postma, 2011; Crowther et al., 2000; Hupbach \& Nadel, 2005; Huttenlocher \& Vasilyeva, 2003; Learmonth, Nadel, \& Newcombe, 2002; Learmonth, Newcombe, \& Huttenlocher, 2001; Learmonth, Newcombe, Sheridan, \& Jones, 2008; Lee, Sovrano, \& Spelke, 2012; Lee \& Spelke, 2010, 2011; Lee, Winkler-Rhoades, \& Spelke, 2012; León, Cimadevilla, \& Tascón, 2014; Lew et al., 2000, 2006; Lourenco, Addy, \& Huttenlocher, 2009; Lourenco \& Cabrera, 2015; Mandolesi, Petrosini, Menghini, Addona, \& Vicari, 2009; Merrill et al., 2016; Nardini, Atkinson, \& Burgess, 2008; Nardini et al., 2006; Nardini, Jones, et al., 2008; Negen, Heywood-Everett, Roome, \& Nardini, 2018; Newcombe, Ratliff, Shallcross, \& Twyman, 2010; Pentland, Anderson, Dye, \& Wood, 2003; Piccardi et al., 2015; RodriguezAndres, Juan, Mendez-Lopez, Perez-Hernandez, \& Lluch, 2016; Rodriguez-Andres, Mendez-Lopez, Juan, \& Perez-Hernandez, 2018; Ruggiero et al., 2016; Yang et al., 2019; Yousif \& Lourenco, 2017), while one article used coincident landmarks (Kaufman \& Needham, 2011). The combination of the two type of
505

506

507

508

509

510 


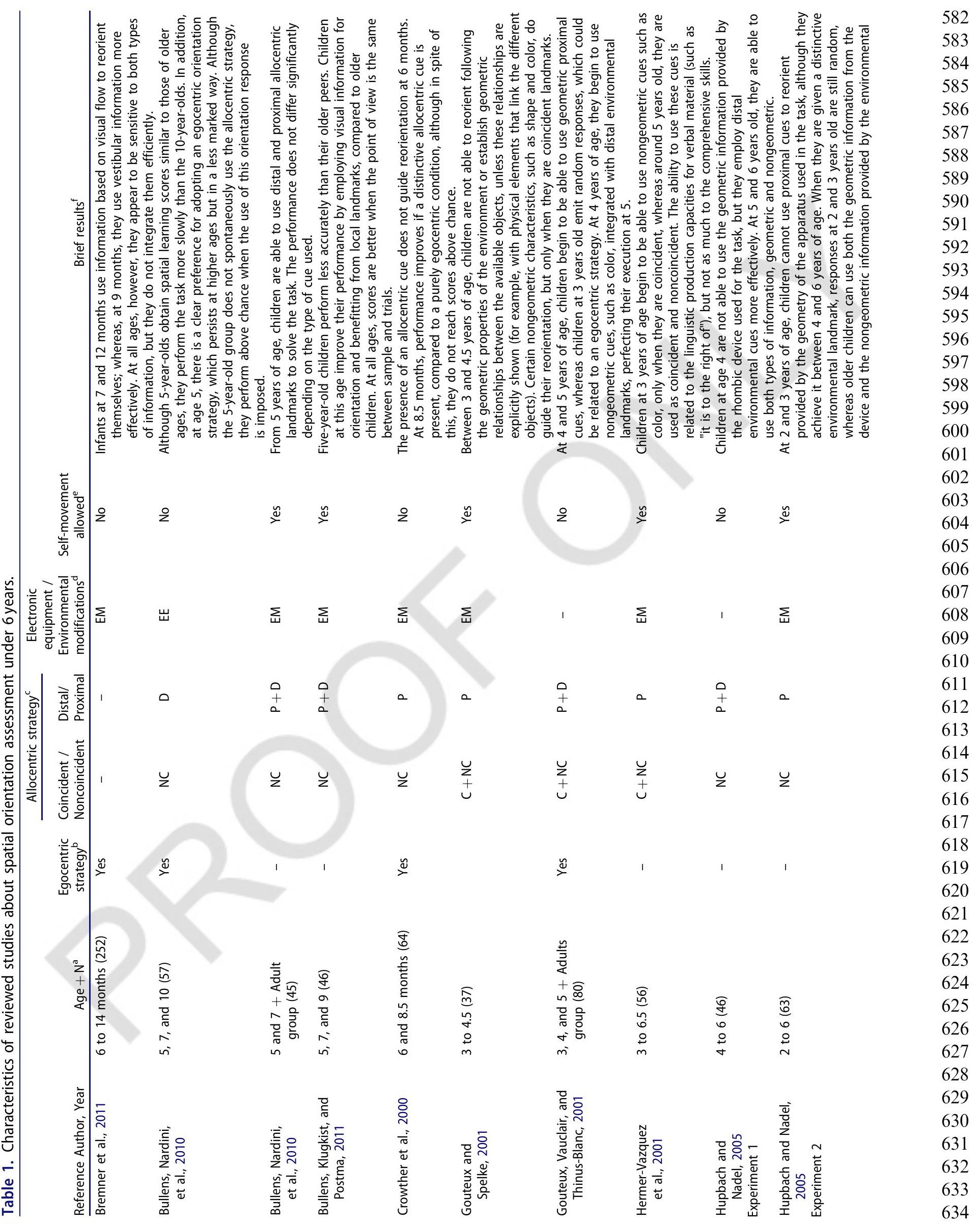




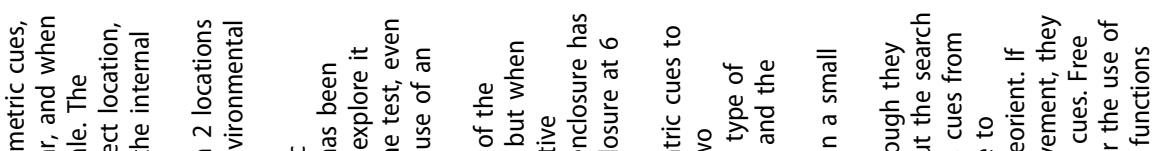

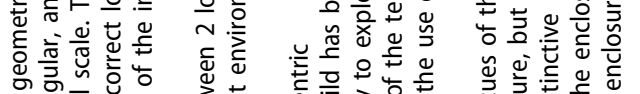

б̄

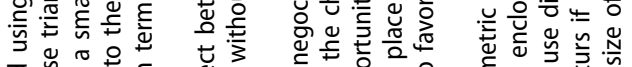

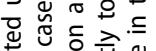

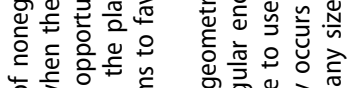

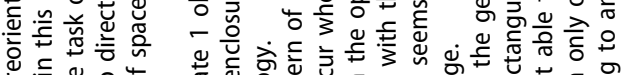

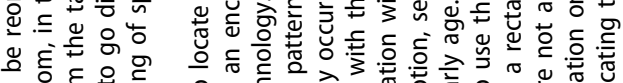

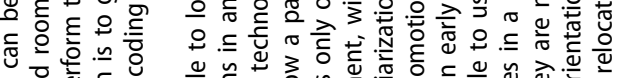

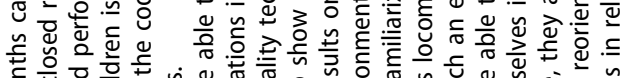

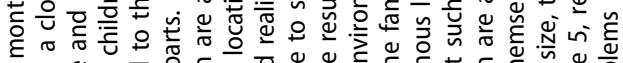

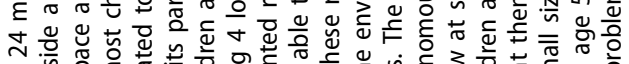

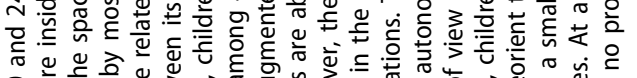

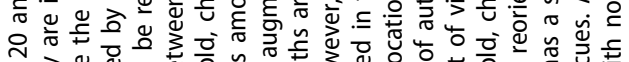

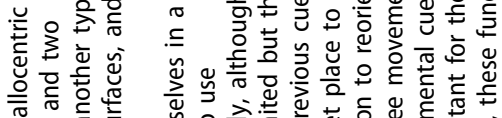

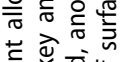

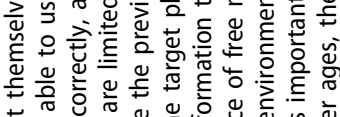

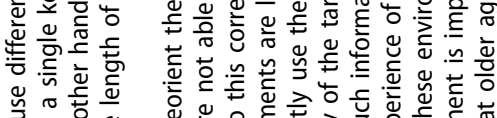

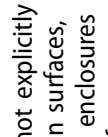

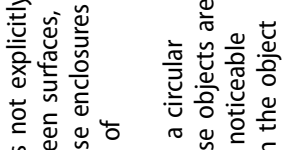

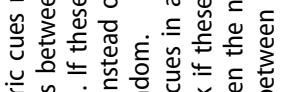

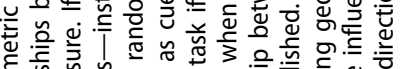

है.

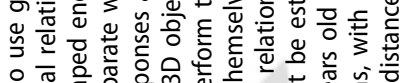

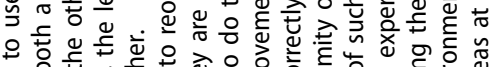

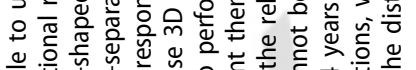

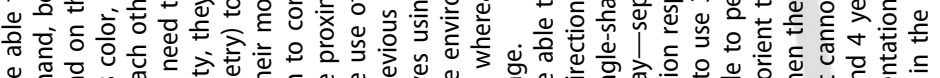

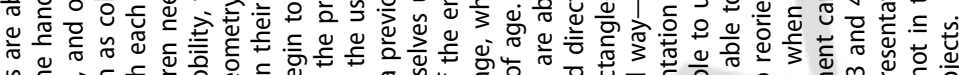

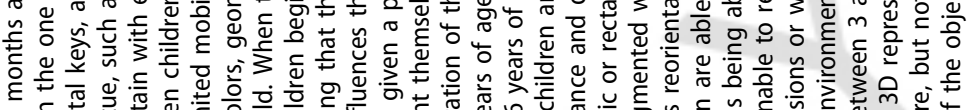

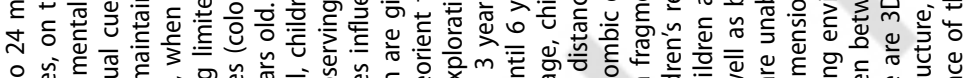

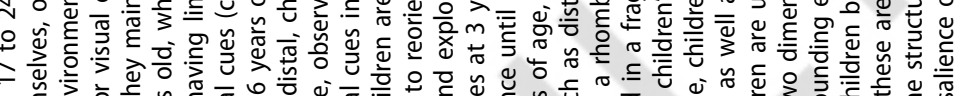

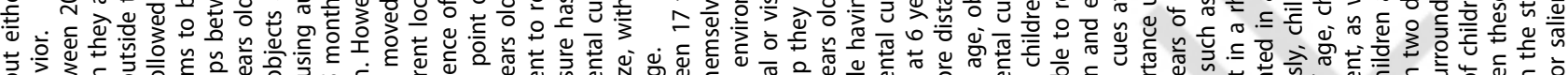

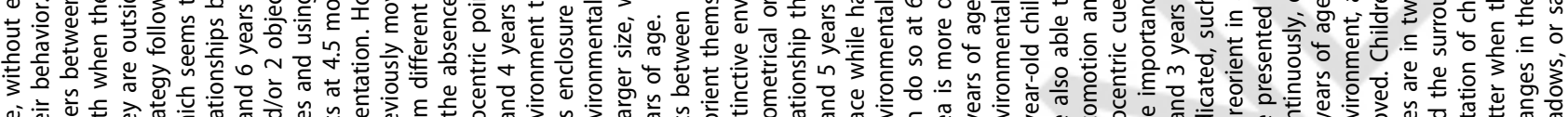

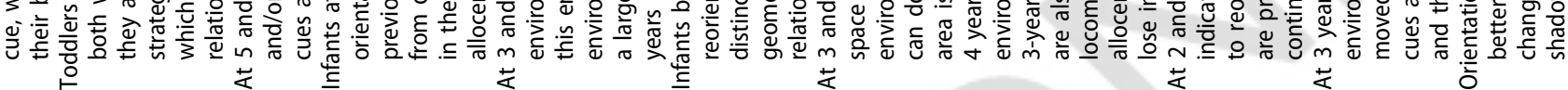

658

659

660

661

662

663

66

665

666

667

668

669

670

671

672

673

674

675

676

677

678

679

680

681

682

68.

684

685

686

687
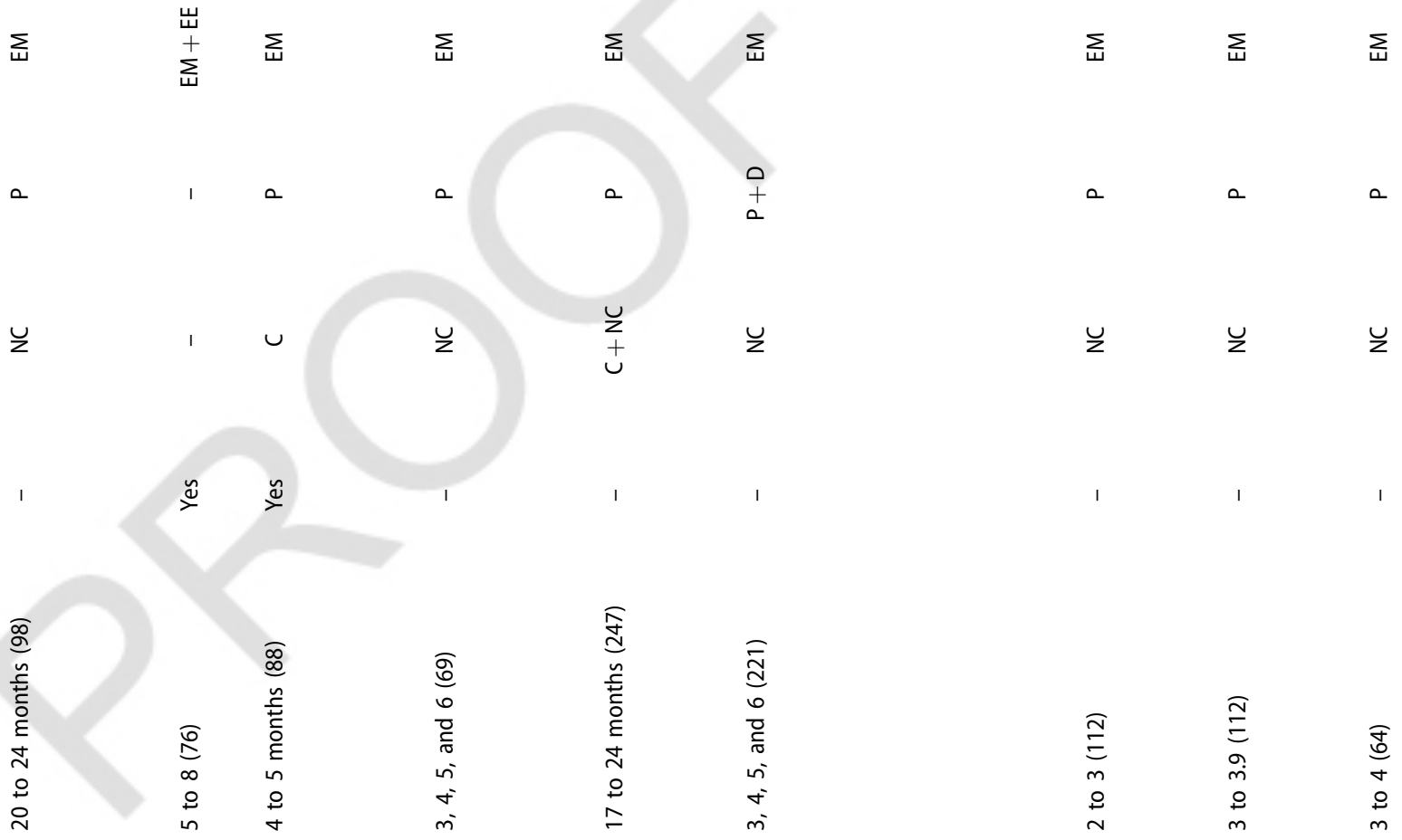

688

689

690

691

692

693

694

695

696

697

698

699

700

701

702

703

704

705

706

707

708

709

710

711

712

713

714

715

716

717

718

719

720

721

722

723

724

725

726

727

728

729

730

731

732

733

734

735

736

737

738

739

740 


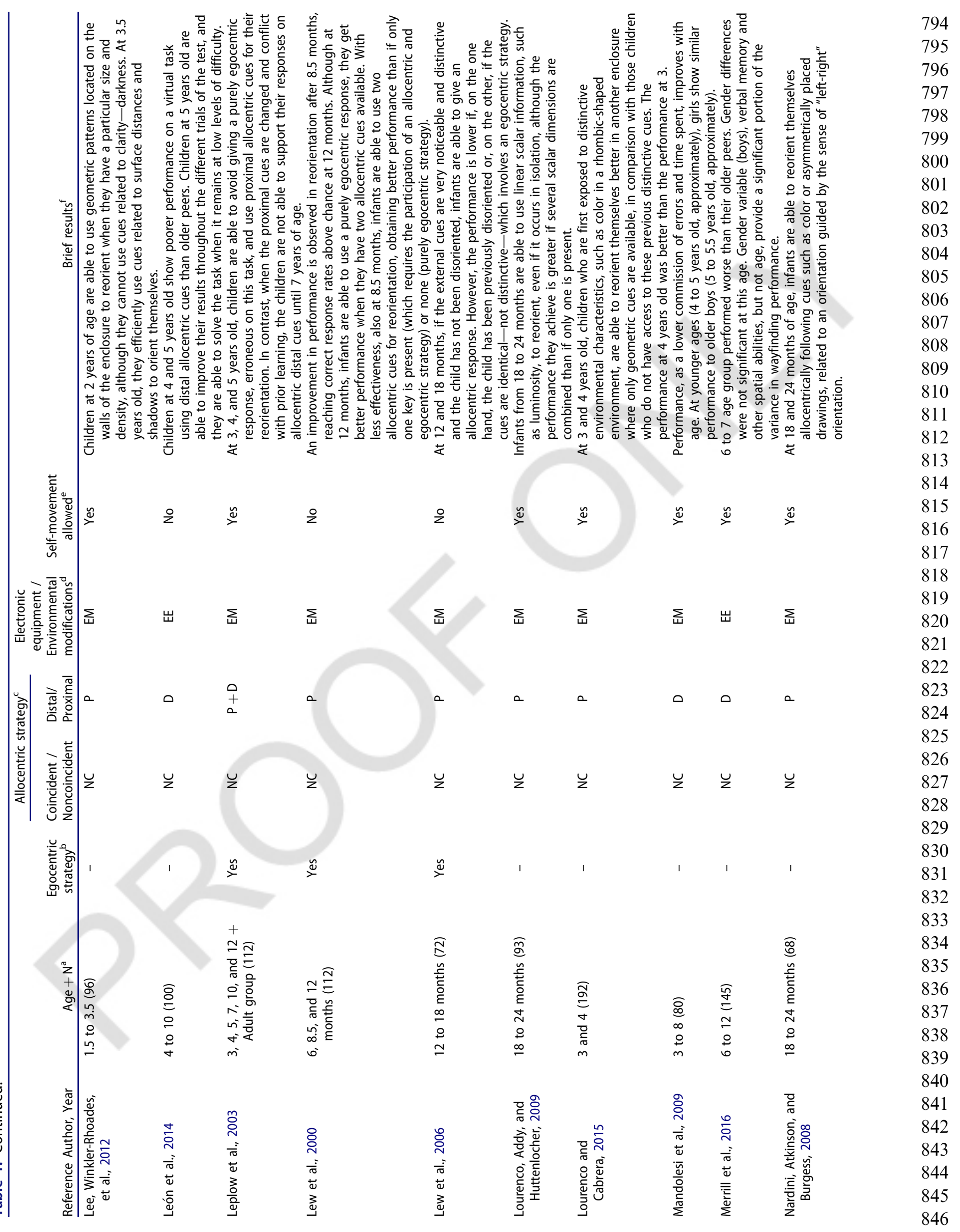


847

848

849

850

851

852

853

854

855

856

857

858

859

860

861

862

863

864

865

866

867

868

869

870

871

872

873

874

875

876

878

879

880

881

882

883

884

885

886

887

888

889

890

891

892

893

894

895

896

897

898

899

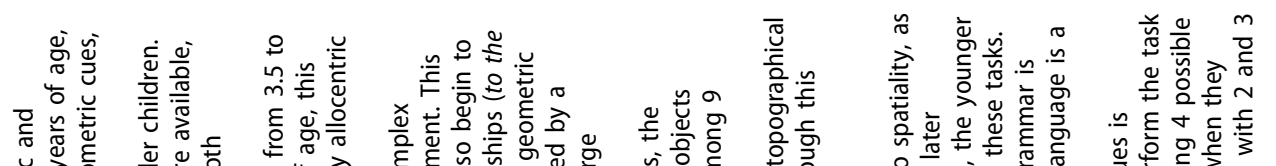

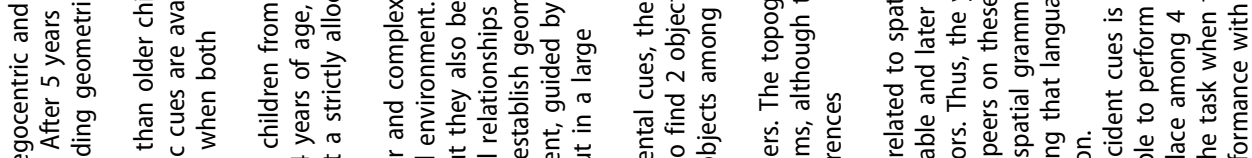

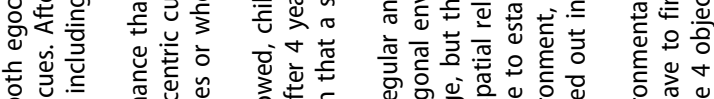

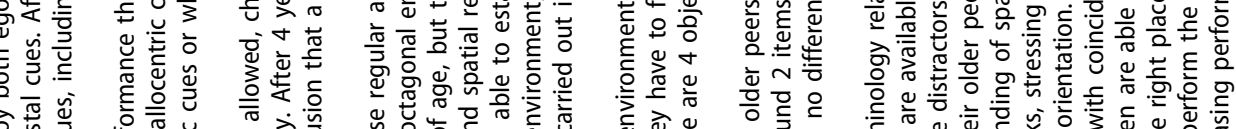

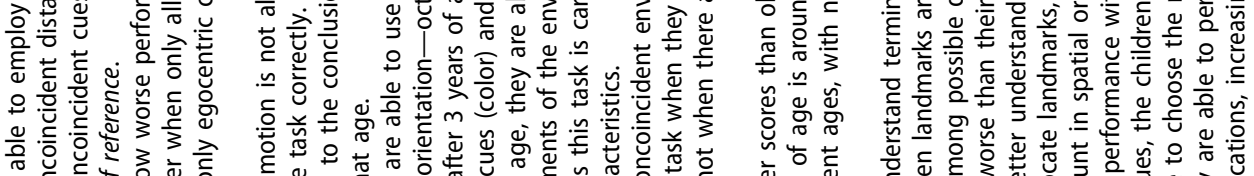

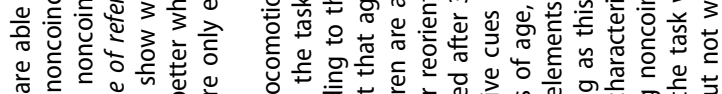

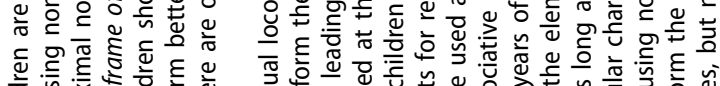

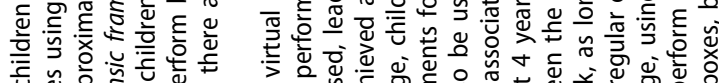

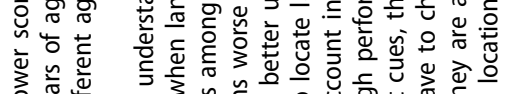

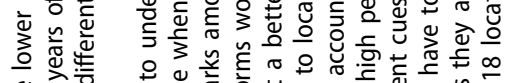

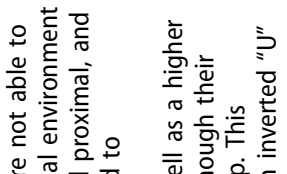

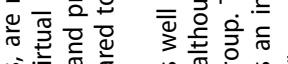

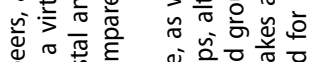

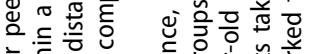

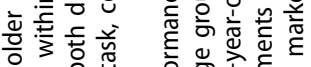

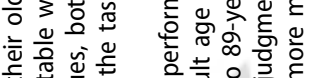

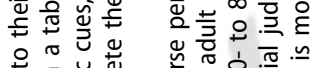

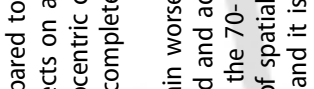

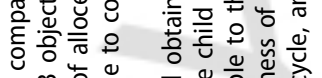

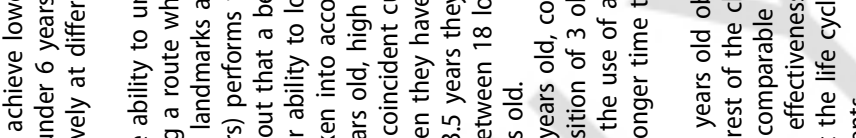

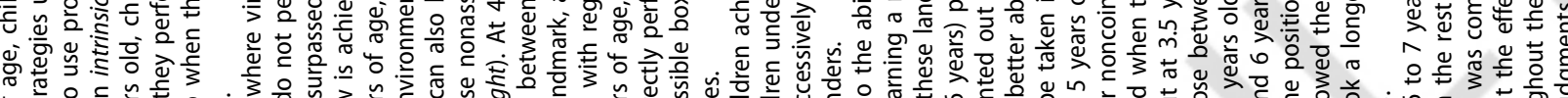

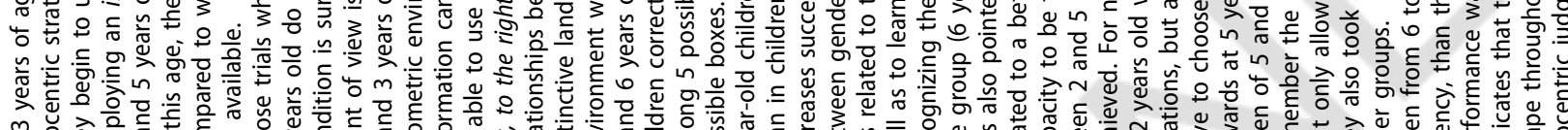

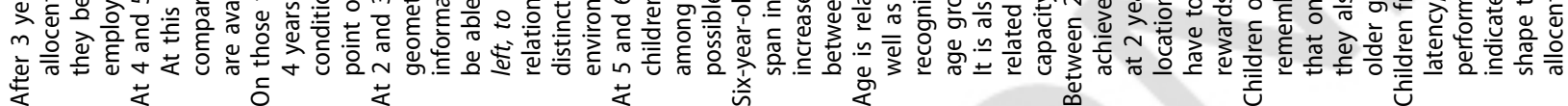
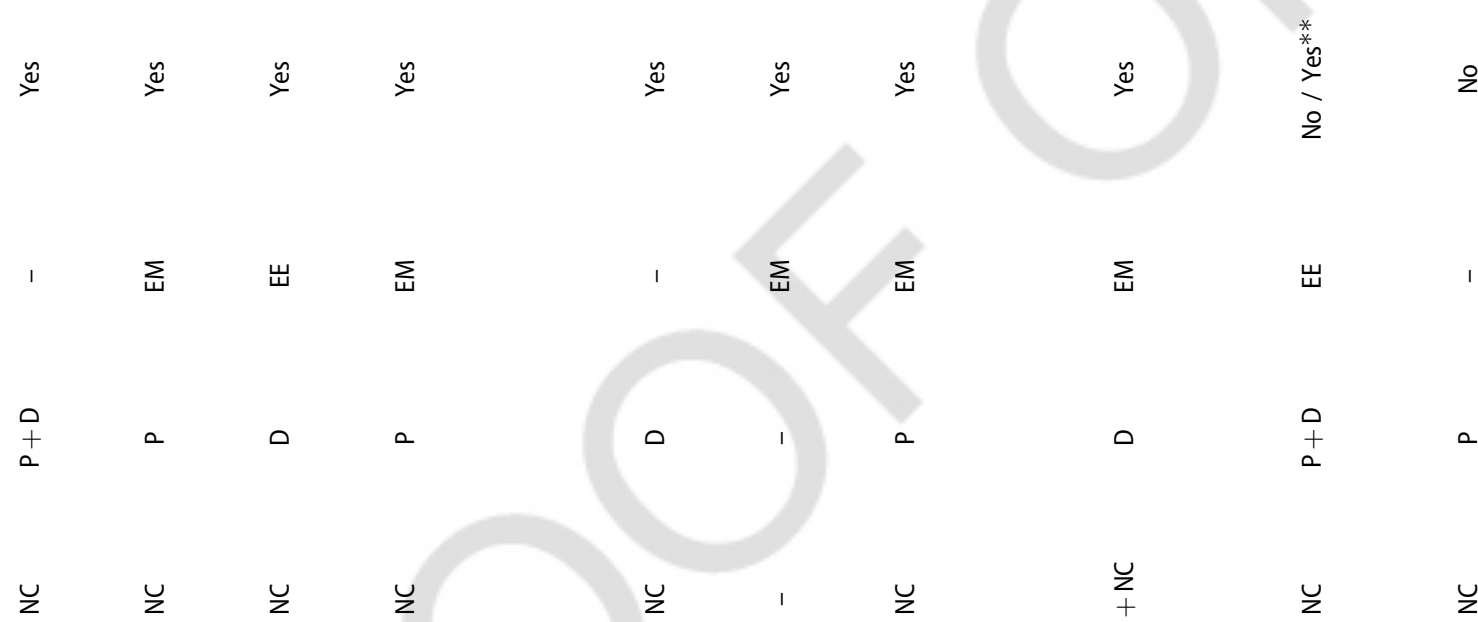

$\sum_{i} \sum_{w}$
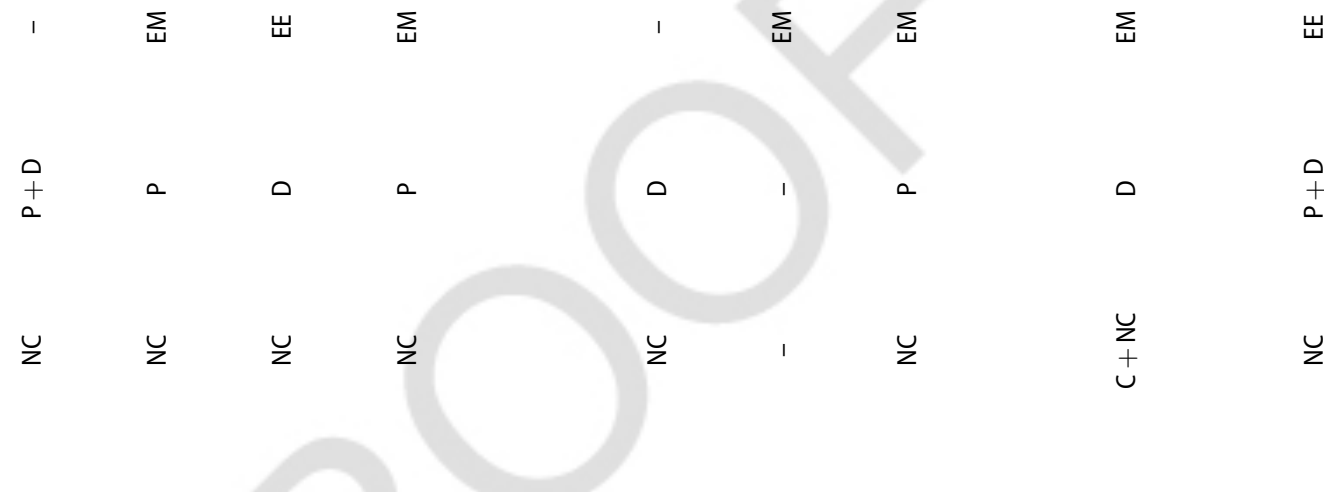

$\stackrel{\varpi}{\check{\Sigma}}$

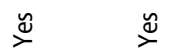

$\stackrel{u}{+}$

z

z

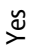

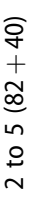

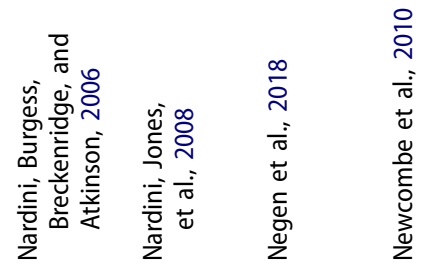

ละ 900

901

902

903

904

905

906

907

908

909

910

911

912

913

914

915

916

917

918

919

920

921

922

923

924

925

926

927

928

929

930

931

932

933

934

935

936

937

938

939

940

941

942

943

944

945

946

947

948

949

950

951

952 


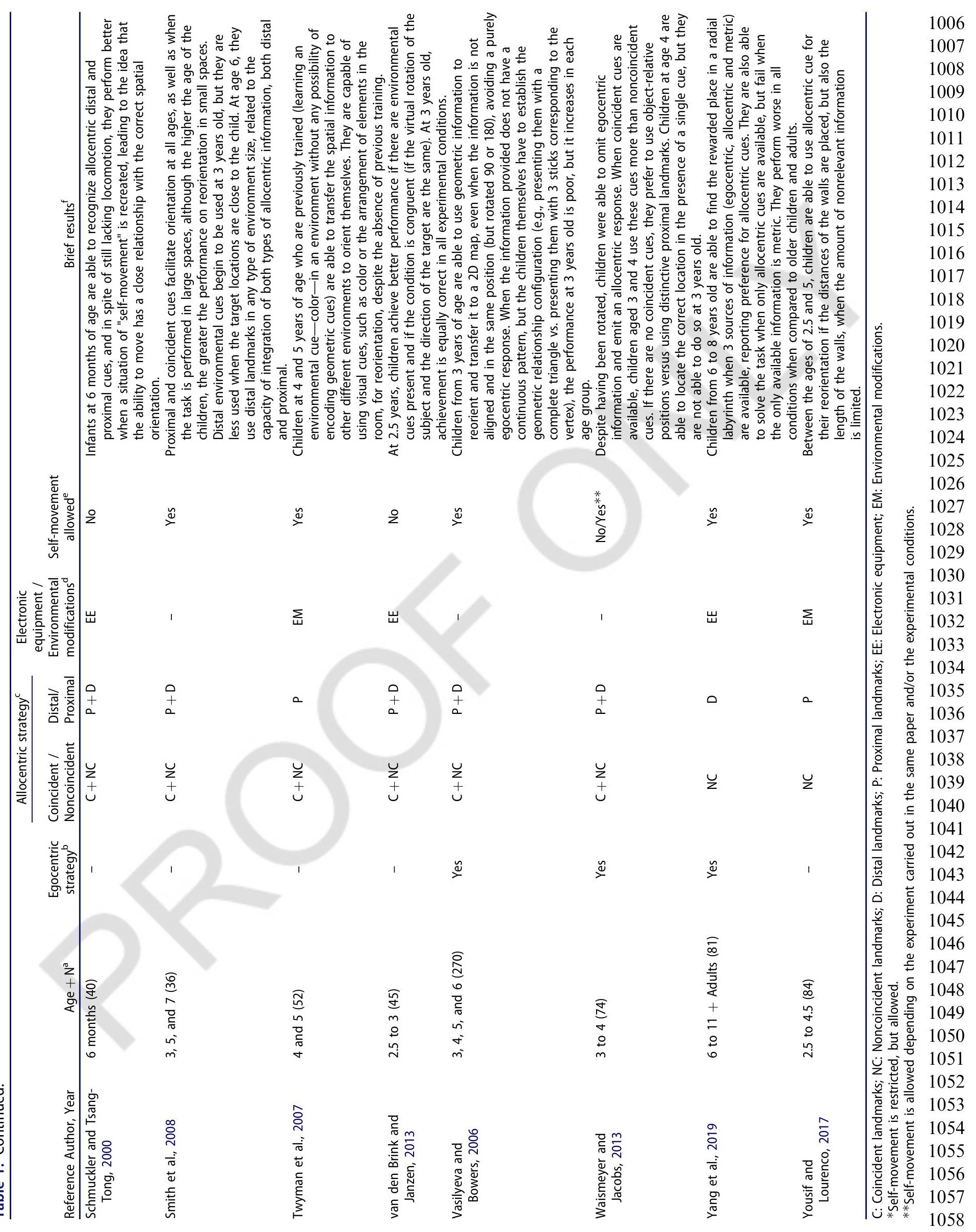


cues was found in 11 articles (Gouteux \& Spelke, 2001; Gouteux et al., 2001; Hermer-Vazquez, Moffet, \& Munkholm, 2001; Ribordy, Jabès, Banta Lavenex, \& Lavenex, 2013; Ribordy Lambert, Lavenex, \& Banta Lavenex, 2017; Schmuckler \& Jewell, 2007; Smith et al., 2008; Twyman, Friedman, \& Spetch, 2007; van den Brink \& Janzen, 2013; Vasilyeva \& Bowers, 2006; Waismeyer \& Jacobs, 2013).

Twenty-two studies used proximal cues (Crowther et al., 2000; Gouteux \& Spelke, 2001; Hermer-Vazquez et al., 2001; Huttenlocher \& Vasilyeva, 2003; Kaufman \& Needham, 2011; Learmonth et al., 2002, 2001; Lee, Sovrano, et al., 2012; Lee \& Spelke, 2010, 2011; Lee, Winkler-Rhoades, et al., 2012; Lew et al., 2000, 2006; Lourenco et al., 2009; Lourenco \& Cabrera, 2015; Nardini, Atkinson, et al., 2008; Nardini, Jones, et al., 2008; Newcombe et al., 2010; Piccardi et al., 2015; Ruggiero et al., 2016; Twyman et al., 2007; Yousif \& Lourenco, 2017), while those using both proximal and distal cues in the tasks were 14 (Bullens, Nardini, et al., 2010; Bullens et al., 2011; Gouteux et al., 2001; Hupbach \& Nadel, 2005; Learmonth et al., 2008; Leplow et al., 2003; Nardini et al., 2006; RodriguezAndres et al., 2016, 2018; Schmuckler \& Jewell, 2007; Smith et al., 2008; van den Brink \& Janzen, 2013; Vasilyeva \& Bowers, 2006; Waismeyer \& Jacobs, 2013) and those using only distal cues were 9 (Bullens, Iglói, et al., 2010; León et al., 2014; Mandolesi et al., 2009; Merrill et al., 2016; Negen et al., 2018; Pentland et al., 2003; Ribordy et al., 2013; Ribordy Lambert et al., 2017; Yang et al., 2019).

The proximal cues usually used in the studies were traffic cones (Bullens, Nardini et al., 2010), small colored or geometrical wood pieces (Bullens, Klugkist, \& Postma, 2011; Ruggiero et al., 2016), paper lanterns (Crowther et al., 2000; Lew et al., 2000), rhomboid tabletop (Hupbach \& Nadel, 2005), cylindrical figures (Huttenlocher \& Vasilyeva, 2003), colored paper squares (Learmonth et al., 2002), bookshelves and doors (Learmonth et al., 2001), columns (Lee, Winkler-Rhoades, et al., 2012), sculpture objects (Lew et al. 2006), led light figures (Nardini, Jones, et al., 2008), object pictures (Piccardi et al., 2015), toys (Waismeyer \& Jacobs, 2013), colored or geometrically printed walls or curtains (Learmonth et al., 2008; Lourenco, Addy, \& Huttenlocher, 2009; Lourenco \& Cabrera, 2015; Nardini, Atkinson, \& Burgess, 2008; Twyman et al., 2007), plastic boxes or colored glasses (Learmonth et al., 2001; Schmuckler \& Jewell, 2007; Smith et al., 2008), as well as the use of the environment's own geometry as an allocentric cue (Gouteux et al., 2001; Lee, Sovrano, \& Spelke, 2012: Yousif \&
Lourenco, 2017). The distal cues used were shaped neon lights (Bullens, Nardini et al., 2010), colored papers (Bullens et al., 2011), colored curtains and cardboards (Gouteux \& Spelke, 2001; Hupbach \& Nadel, 2005), and furniture (Gouteux et al., 2001; Ribordy et al., 2013; Ribordy Lambert et al., 2017; Vasilyeva \& Bowers, 2006). Studies assessing performance in outdoor tasks normally used distal cues, usually trees, swings, benches, water towers, and so forth (Mandolesi et al., 2009; Smith et al., 2008). Virtual tasks usually created natural environments using landmarks such as trees, mountains, rocks, or buildings, but pictures, doors, and windows were also used as landmarks when domestic environments were used (Bullens, Iglói, et al., 2010; León et al., 2014; Merrill et al., 2016; Negen et al., 2018; van den Brink \& Janzen, 2013; Yang et al., 2019).

Studies using proximal and distal cues are based on the presence of both cues at the same time (Gouteux et al., 2001; Hupbach \& Nadel, 2005; RodriguezAndres et al., 2016, 2018; Vasilyeva \& Bowers, 2006). These studies used different experimental conditions in order to compare children's performance when an additional distinctive cue was used (Bullens et al, 2011; Learmonth et al., 2008; Smith et al., 2008; van den Brink \& Janzen, 2013). The studies also assessed whether changing the position of one of the cues, while the others remain in the same place, has any effect on performance (Bullens, Nardini et al., 2010; Leplow et al., 2003; Nardini et al., 2006; Schmuckler \& Jewell, 2007; Waismeyer \& Jacobs, 2013). Thus, in these studies the experimental conditions were designed to create conflict between different types of landmarks, assess how an extra landmark can improve performance, or explore how children can use both proximal and distal landmarks at the same time.

\section{Locomotion, equipment and technologies in spatial orientation}

Thirty-eight of the 49 articles analyzed in the present review include all or some experimental conditions that require the child to move in order to solve the task (Bullens, Iglói, et al., 2010; Bullens, Nardini et al., 2010; Gouteux \& Spelke, 2001; Hermer-Vazquez et al., 2001; Hupbach \& Nadel, 2005; Huttenlocher \& Vasilyeva, 2003; Juan et al., 2014; Learmonth et al., 2002, 2001, 2008; Lee, Sovrano, et al., 2012; Lee \& Spelke, 2011, 2010; Lee, Winkler-Rhoades, et al., 2012; Leplow et al., 2003; Lourenco et al., 2009; Lourenco \& Cabrera, 2015; Mandolesi et al., 2009; Merrill et al., 2016; Nardini, Atkinson, et al., 2008; Nardini et al., 
2006; Nardini, Jones, et al., 2008; Negen et al., 2018; Newcombe et al., 2010; Pentland et al., 2003; Piccardi, Leonzi, et al., 2014; Piccardi, Palermo, et al., 2014; Piccardi et al., 2015; Ribordy et al., 2013; Ribordy Lambert et al., 2017; Rodriguez-Andres et al., 2016, 2018; Smith et al., 2008; Twyman et al., 2007; Vasilyeva \& Bowers, 2006; Waismeyer \& Jacobs, 2013; Yang et al., 2019; Yousif \& Lourenco, 2017). Spatial orientation requires displacement around the navigational environment. For this reason, allowing children to move around during the tasks is a relevant issue. The tasks where the child does not move are mainly for the youngest children, aged 18 months or below (Crowther et al., 2000; Kaufman \& Needham, 2011; Lew et al., 2000, 2006; Schmuckler \& Tsang-Tong, 2000), where even if the infants do not move by themselves, they are usually moved by their caregivers. For older children, the tasks where self-movement is not allowed are those that use computers or other types of technologies (Bullens, Iglói, et al., 2010; León et al., 2014; van den Brink \& Janzen, 2013), one task related to spatial judgments from a static position (Ruggiero et al., 2016), and one task carried out in a real environment as well (Gouteux et al., 2001). However, this does not occur in all the tasks that include technological equipment. For example, some studies use a virtual reality task in which the children are required to move (Negen et al., 2018), using active mobility interfaces during the virtual task, such as a platform for their feet and a car steering wheel (Rodriguez-Andres et al., 2016), and employing augmented reality in a real environment that allows them to use their own locomotion (Juan et al., 2014).

A total of 37 articles design spatial orientation tasks that happen in real environments (Bullens, Nardini et al., 2010; Bullens et al., 2011; Crowther et al., 2000; Gouteux \& Spelke, 2001; Gouteux et al., 2001; Hermer-Vazquez et al., 2001; Hupbach \& Nadel, 2005; Huttenlocher \& Vasilyeva, 2003; Juan et al., 2014; Kaufman \& Needham, 2011; Learmonth et al., 2001, 2008, 2002; Lee, Sovrano, et al., 2012; Lee \& Spelke, 2010, 2011; Lee, Winkler-Rhoades, et al., 2012; Leplow et al., 2003; Lew et al., 2000, 2006; Lourenco et al., 2009; Lourenco \& Cabrera, 2015; Mandolesi et al., 2009; Nardini, Atkinson, et al., 2008; Nardini, Jones, et al., 2008; Newcombe et al., 2010; Pentland et al., 2003; Piccardi, Leonzi, et al., 2014; Piccardi, Palermo, et al., 2014; Piccardi et al., 2015; Ribordy et al., 2013; Ribordy Lambert et al., 2017; Ruggiero et al., 2016; Smith et al., 2008; Twyman et al., 2007; Vasilyeva \& Bowers, 2006; Yousif \& Lourenco, 2017). From these, 31 of them require some type of environmental modifications. These studies are mainly carried out in empty rooms or enclosures with different shapes (mainly square, rectangular or round enclosures, but also rhomboid, octagonal or radial labyrinths) and use curtains or panels to avoid access to distal cues (Bullens, Nardini et al., 2010; Bullens et al., 2011; Crowther et al., 2000; Gouteux \& Spelke, 2001; Hermer-Vazquez et al., 2001; Hupbach \& Nadel, 2005; Huttenlocher \& Vasilyeva, 2003; Juan et al., 2014; Kaufman \& Needham, 2011; Learmonth et al., 2008, 2002, 2001; Lee, Sovrano, et al., 2012; Lee \& Spelke, 2010, 2011; Lee, Winkler-Rhoades, et al., 2012; Leplow et al., 2003; Lew et al., 2000, 2006; Lourenco et al., 2009; Lourenco \& Cabrera, 2015; Nardini, Atkinson, et al., 2008; Nardini, Jones, et al., 2008; Newcombe et al., 2010; Piccardi, Leonzi, et al., 2014; Piccardi, Palermo, et al., 2014; Piccardi et al., 2015; Ribordy et al., 2013; Ribordy Lambert et al., 2017; Twyman et al., 2007; Yousif \& Lourenco, 2017). We also found some tasks that use the elements commonly present in the room (furniture, doors, windows, etc.) as allocentric landmarks for reorientation (Gouteux et al., 2001; Hupbach \& Nadel, 2005; Nardini et al., 2006; Pentland et al., 2003; Vasilyeva \& Bowers, 2006), thus requiring fewer environmental modifications than those that create specific enclosures. Additionally, two of these real-world based tasks are performed in outdoor environments (Mandolesi et al., 2009; Smith et al., 2008), although one of them also requires specific equipment (Mandolesi et al., 2009).

Regarding technology-based tasks, nine of the studies require some electronic equipment to be carried out, needing one of them a television and videotapes (Schmuckler \& Tsang-Tong, 2000), a computer (Bullens, Iglói, et al., 2010; León et al., 2014; Merrill et al., 2016; van den Brink \& Janzen, 2013; Yang et al., 2019), augmented reality technology (Juan et al., 2014; Rodriguez-Andres et al., 2016, 2018), and virtual reality technology (Negen et al., 2018).

\section{Development of spatial orientation abilities}

As shown in Table 1 , between 7 and 12 months of age, infants appear to be sensitive to changes in visual flow and vestibular information, sources of information that are vital for proper spatial orientation (Bremner et al., 2011). Between about 4.5 months (Kaufman \& Needham, 2011) and 6 months of age (Schmuckler \& Jewell, 2007), infants seem to be able to show an orientation response employing coincident cues, but this result only appears if infants have previously been moved by the environment and in 
combination with egocentric information (Kaufman \& Needham, 2011) or if this movement has been simulated (Schmuckler \& Jewell, 2007).

Conversely, 6-month old infants do not manage to reorient themselves following a distinctive noncoincident allocentric landmark (Crowther et al., 2000). At 8.5 months of age, infants improve their orientation performance if there is one noncoincident landmark (Crowther et al., 2000) or two noncoincident landmarks (Lew et al., 2000); that is, their performance is better following an allocentric framework, rather than a purely egocentric framework. Moreover, although at 12 months old they are able to respond correctly when the only information available is self-centered, their ability to orient themselves improves if noncoincident allocentric cues are present (Lew et al., 2000). From this age and up to 18 months, toddlers are able to orient themselves toward distinctive and noncoincident allocentric landmarks as long as they have not previously been disoriented (Lew et al., 2006). Furthermore, at these ages, they manage to respond egocentrically, but with less success compared to an allocentric response (Lew et al., 2006). From 18 months to 2 years old, the ability to give allocentric responses after being disoriented improves greatly. Toddlers are be able to use different types of cues: geometric and/or visual cues, such as the color or length of the surface of the environment (Learmonth et al., 2001), cues based on linear scalar information, such as luminosity (Lourenco et al., 2009), and colorbased cues (Nardini, Atkinson, et al., 2008), all of which are noncoincident landmarks. In addition, at these ages they are able to code the spatial relationships in the environment and use this information to reorient themselves (Huttenlocher \& Vasilyeva, 2003) and establish spatial relationships based on a sense of "left-right" (Nardini, Atkinson, et al., 2008).

From 2 to 3 years old, children continue to use different types of noncoincident allocentric cues based on geometry, such as distances and relationships between the surfaces of the enclosures (Lee, Sovrano, et al., 2012), differential patterns with specific sizes and densities on the walls of the enclosure (Lee, Winkler-Rhoades, et al., 2012), and complex and regular octagonal environments (Newcombe et al., 2010). Moreover, starting from these ages, children will not present difficulties in reorienting following coincident landmarks (Ribordy et al., 2013; Ribordy Lambert et al., 2017) or reorienting on virtual tasks, guided by coincident and/or noncoincident landmarks (van den Brink \& Janzen, 2013). However, they will still present problems in reorienting themselves in certain specific geometrical environments, such as rhomboids, despite having distinctive allocentric cues (Hupbach \& Nadel, 2005), or in using landmarks related to clarity-darkness (Lee, Winkler-Rhoades, et al., 2012). Up to this point, all the landmarks used for reorientation have been proximal, but after 2 years of age, children seem to start to use distal landmarks for the first time to locate an object among four possible locations (Ribordy et al., 2013; Ribordy Lambert et al., 2017).

From 3 to 4 years of age, children can use proximal and noncoincident cues related to superficial distances and shadows to orient themselves (Lee, WinklerRhoades, et al., 2012), subtle geometric cues on surfaces in $3 \mathrm{D}$ (Lee \& Spelke, 2011), and the distance and length of the surrounding walls (Yousif \& Lourenco, 2017). However, they are not yet able to establish these geometric relationships between the elements of space when they are disconnected or separated from each other. Instead, these relationships have to be explicitly shown with panels or physical elements that join the different objects (Gouteux \& Spelke, 2001; Vasilyeva \& Bowers, 2006). It also seems that at these ages, nongeometric proximal cues such as color (Hermer-Vazquez et al., 2001; Lourenco \& Cabrera, 2015; Newcombe et al., 2010) acquire greater importance, improving performance if these colored cues are coincident (Hermer-Vazquez et al., 2001) and having a subsequent facilitating effect on reorientation in the absence of landmarks (Lourenco \& Cabrera, 2015). At these ages, children seem to take advantage of "leftright" spatial relationships for reorientation as well (Newcombe et al., 2010).

Although the preference for using coincident and/ or proximal cues continues if they are available (Waismeyer \& Jacobs, 2013), at 3 years of age they can use distal allocentric landmarks more successfully (Nardini et al., 2006; Smith et al., 2008). However, not all studies agree with these results at such an early age (Leplow et al., 2003), where children obtain better performance using proximal rather than distal cues. Another issue that seems to improve allocentric orientation at these ages is allowing locomotion and selfmovement, either real (Leplow et al., 2003) or virtually simulated (Negen et al., 2018). In fact, when the movement of 3-year-old children is not allowed, findings show that they are not able to use geometric proximal cues, but only egocentric responses (Gouteux et al., 2001). Other relevant variables that promote the use of allocentric orientation are the three-dimensionality of the target locations-instead of two-dimensionality-and maintaining the stability of the spatial relationships between these possible 
locations and the surrounding environment (Lee \& Spelke, 2010), as well as the size of the environment or place where the task is carried out because it seems that at these ages they are not able to use distinctive allocentric cues when the task is performed in small rectangular enclosures (Learmonth et al., 2002).

The aforementioned aspects continue to improve from 4 to 5 years of age. At these ages, children can establish spatial relationships between separate objects when spatial relationships between the objects are not explicitly shown, progressively improving with age (Vasilyeva \& Bowers, 2006) and guided by a distinctive and proximal landmark in large environments with regular characteristics (Newcombe et al., 2010). In addition, they seem to use proximal geometric landmarks with greater efficacy (Gouteux et al., 2001). However, they are still unable to integrate them with distal allocentric information (Hupbach \& Nadel, 2005).

Regarding the use of distal information, some authors report a predominance of its use in some cases (Hupbach \& Nadel, 2005), whereas others describe better performance with proximal cues (Leplow et al., 2003), but an inability to use distal information until more advanced ages persist. The use of nongeometric landmarks, such as color, which is reported to be initially used in combination with other distal environmental cues, also becomes increasingly important, as children may reorient themselves by nongeometric properties by forming direct associations between a hidden object, the distinctive color of the landmark and environmental stimuli (Gouteux et al., 2001). In addition, previous spatial learning based on color cues can be transferred and generalized to untrained or unexplored environments (Twyman et al., 2007). Although at these ages children can integrate different types of environmental cues, such as proximal nongeometric landmarks with distal stimuli (Gouteux et al., 2001), some authors disagree on this point. They argue that better performance is found at these ages only in the presence of allocentric landmarks, compared to the presence of both egocentric and allocentric cues (Nardini, Jones, et al., 2008). Thus, children at these ages seem to perform better using allocentric cues (based on landmarks) than egocentric cues (based on auto-locomotion), but in addition, the performance of these children is worse when both types of cues are available, indicating that they are not yet able to integrate them, unlike adult subjects (Nardini, Jones, et al., 2008). Thus, it could happen that when children are provided with a unique distinctive and proximal cue, as well as when self-locomotion is not required and there is no disorientation procedure, they can associate this cue with the environment (Gouteux et al., 2001). On the other hand, they would still show difficulties in more complex tasks, where the child must simultaneously take into account their own movements and the available environmental landmarks (Nardini, Jones, et al., 2008). Limitations in self-movement during spatial orientation tasks seem to be less relevant after 4 years old: from these ages on, children can use more distal geometric and nongeometric cues to reorient successfully, despite limiting their freedom of movement in small enclosures (Learmonth et al., 2008). It also seems that the absence of movement during a virtual task does not impede reorientation based on distal allocentric cues (Negen et al., 2018). Although spatial orientation studies in children do not usually find gender differences among participants, on an allocentric task that uses distal landmarks, at younger ages (4 to 5 years) girls show a similar performance to that of older boys ( 5 to 5.5 years) (Mandolesi et al., 2009).

From age 5 onward, children's orientation skills reach high performance levels. At these ages, children are less limited by the size of the enclosure where the task is carried out, still finding better orientation in larger enclosures at 5 years old (Learmonth et al., 2002), but the size of the area does not affect performance at around 6 years of age (Learmonth et al., 2002, 2008; Smith et al., 2008). There are no longer difficulties in the management of proximal geometric cues (Nardini et al., 2006) or a preference for the use of proximal cues versus the use of distal cues (Bullens, Nardini et al., 2010; Hupbach \& Nadel, 2005), although the use of proximal or local landmarks are associated with greater success in reorientation (Bullens et al., 2011; Smith et al., 2008). Moreover, when there is a choice between one strategy or the other, they perform better on virtual tasks with the egocentric strategy than with the allocentric one at these ages (Bullens, Iglói, et al., 2010).

Six-year-old children show an improvement in their spatial learning when they navigate in virtual environments with low difficulty levels and using distal cues (León et al., 2014) Also, on these tasks, 6year-old boys perform better than girls (León et al., 2014). Moreover, at this age, some authors found that children can successfully utilized process of integration across different scales, when distal landmarks need to be integrated with proximal locations (Smith et al., 2008). It seems that they code locations at both proximal and distal spatial scales in allocentric coordinates (Smith et al., 2008). However, other authors 
found that this is not possible until 7 years of age (Leplow et al., 2003). However, the procedures of these studies are different. In Smith et al. (2008), the tracks always remained in the same place and the task was done in an open environment. In Leplow et al. (2003), the proximal landmarks were rotated coming into conflict with the distal ones and the task took place in a circular and closed environment. From these ages, children can orient themselves with relative efficacy; therefore, the interest of research is to test how much spatial information in the environment they are capable of retaining. The results from studies on this topic are varied. For example, using distal cues, some studies have found that 5 -year-old children are able to remember between 2 and 3 locations among 18 possible locations (Ribordy et al., 2013; Ribordy Lambert et al., 2017), but others have found that at 5 and 6 years of age, they only remember 2 locations among 5 possible locations, but they are not able to remember 4 locations among 9 possible locations (Pentland et al., 2003). When both distal and proximal landmarks are available, children are not able to remember the position of 3 objects within a virtual environment at 5 and 6 years old (RodriguezAndres et al., 2016, 2018).

Exclusively using the egocentric orientation, 5 and 6 -year-old children were able to locate 1 object from 2 locations and 2 objects among 4 locations (Juan et al., 2014). Therefore, we can conclude that the number of possible locations does not seem to be as relevant as the amount of information to be remembered. Thus, it seems that in children under 6 years old, the topographical span, that is, how much spatial information we can remember in the surrounding environment, is around 2 items, and this increases successively at different ages (Piccardi, Leonzi, et al., 2014; Piccardi et al., 2015). In addition, from the age of 6 , progressive knowledge of space-related vocabulary and grammar will help to improve landmarkbased performance throughout childhood (Piccardi et al., 2015). It is also worth mentioning that from age 6 to 7 , the management of egocentric and allocentric spatial strategies will continue to improve during childhood, adolescence, and adulthood, and then progressively decrease in the elderly (Ruggiero et al., 2016). In addition, from these ages, it has been possible to verify that other variables such as verbal memory and spatial skills influence performance in allocentric orientation (Merrill et al., 2016). Although it has been demonstrated that children can effectively use allocentric cues at this age, they are not yet able to use metric information, such as the length of a corridor, to reorient themselves appropriately (Yang et al., 2019).

\section{Discussion}

We found that the current interest in spatial orientation assessment in preschool children favors allocentric spatial orientation tests instead of egocentric spatial orientation tests. We should also highlight that on many of the assessment tasks, egocentric responses are considered nondesirable for solving the task. Because the egocentric framework is the first to emerge during child development (Acredolo, 1978), most of the researchers have probably focused on finding out when children start using allocentric cues for the first time. However, this lack of interest in the egocentric framework in recent literature is striking, taking into account that following environmental knowledge acquisition (Siegel \& White, 1975), the functional use of landmarks combined with egocentric information - route knowledge-, as well as the creation of mental maps for navigation-survey knowledge-develop later in childhood, not until 7 years old (Overman et al., 1996). All of this indicates that, given that the egocentric framework develops earlier (Acredolo, 1978; Acredolo \& Evans, 1980; Piaget \& Inhelder, 1967), the number of articles that study this frame of reference should be greater at the earliest ages, and they should focus on the study of the allocentric framework as the target ages approach 7 years. Conversely, it is also worth mentioning that the lack of tests to assess the egocentric framework during early childhood complicates the detection of potential alterations in this capacity at these ages, which, as previously mentioned, can help to diagnose children with developmental alterations earlier, especially those with topographical developmental disorientation (Bianchini et al., 2014; Conson et al., 2018; Iaria \& Barton, 2010; Iaria et al., 2009, 2005; Nemmi et al., 2015; Palermo, Piccardi, et al., 2014).

Regarding allocentric tasks, the use of noncoincident cues outpaces the use of coincident cues. Surprisingly, most of the tasks analyzed in the present review that include coincident landmarks assess 3year-old children or older (Gouteux \& Spelke, 2001; Gouteux et al., 2001; Hermer-Vazquez et al., 2001; Smith et al., 2008; Twyman et al., 2007; Vasilyeva \& Bowers, 2006; Waismeyer \& Jacobs, 2013), and only two evaluate coincident cues under the age of two (Kaufman \& Needham, 2011; Schmuckler \& Jewell, 2007). These results are surprising, taking into account that in regular development, coincident cues 
are used before noncoincident landmarks (Acredolo, 1978; Acredolo, Adams, \& Goodwyn, 1984; Rieser, 1979). Therefore, it seems that the natural developmental course of allocentric evaluation would be resolved by including more coincident cues in the first stages of child development and, subsequently, including noncoincident landmarks in the assessment protocols for the evaluation of older children. However, studies have also observed that the preference for coincident cues extends to 4-year-old children (Waismeyer \& Jacobs, 2013), and so it seems reasonable to continue to include these kinds of landmarks in spatial orientation tasks for preschool-aged children. Conversely, the use of proximal cues on allocentric tasks surpasses the use of distal cues. A greater presence of proximal cues is expected on experimental tasks because all the studies that only use this type of landmark are carried out in clearly delimited and closed enclosures, preventing access to remote cues. For this reason, the tasks that are carried out in virtual environments (Bullens et al., 2011; León et al., 2014; Merrill et al., 2016; Rodriguez-Andres et al., 2016, 2018; van den Brink \& Janzen, 2013; Yang et al., 2019), outdoors (Mandolesi et al., 2009; Smith et al., 2008), or in less encapsulated spaces (Nardini et al., 2006; Pentland et al., 2003; Ribordy et al., 2013; Ribordy Lambert et al., 2017; Vasilyeva \& Bowers, 2006) are those that most frequently use distal landmarks. Thus, distal cues are more likely to be included when the task takes place in open environments rather than small rooms.

The examination of the recent literature shows that researchers consider locomotion and/or self-movement to be a relevant variable to take into account for spatial orientation assessment in preschoolers, as almost all of them include it in their experimental tasks. At very early ages, the first locomotor capacities-involved in crawling-have been associated with improvements in spatial abilities (Acredolo et al., 1984). Being able to move around our surrounding environment allows us to know and memorize the space from different perspectives, and this early familiarization allows us to orient ourselves more effectively (Kaufman \& Needham, 2011). Moreover, the studies that manipulate the child's ability to move freely, developing spatial orientation in small enclosures, find worse results in children from 3 to 5 years old when they limit their movement possibilities (Learmonth et al., 2008).

However, because orientation requires movement around the surrounding space, it seems clear that to achieve greater functional validity, it is necessary to allow children's movement even after this age. For this reason, we found that the tasks in which the participant remains static are the ones for infants or toddlers-even if they are old enough to crawl-or those performed on the computer for older ages. Even if the child does not really move, we need to take into account the existence of simulated movement in virtual tasks. Thus, although in many tasks there is no real movement, if this simulated movement is present, compensated by the apparatus or by the virtual task procedure, we should not exclude the influence of real locomotion on their results. However, in our knowledge, the comparison of simulated and real movement was not studied, making it necessary to develop new research in this direction.

Moreover, we need to consider that perceiving our own movements during spatial orientation tasks is not the only relevant information that is present in real environments: vestibular and proprioceptive information, as well as the visual flow, are also present in our daily orientation tasks (Banta Lavenex et al., 2011; Piccardi et al., 2008), and many computerized spatial tasks cannot take this information into account. Therefore, spatial tasks in real environments make it possible to perceive and upload our own movements, take into account the position of the body and the head, and be aware of our own equilibrium, and so forth, so they could be the best option for replicating the usual conditions of spatial orientation, showing greater ecological validity in its evaluation. However, including some new technologies, such as the use of realistic and immersive virtual environments that allow freedom of movement by the user (Negen et al., 2018) or the use of augmented reality in real environments (Juan et al., 2014), could be great future alternatives with high functional relevance because they combine the advantages of using new technologies with the information normally available in spatial orientation.

Functionality or ecological validity of the task is another variable that we need to take into account in order to include some of these tasks in clinical neuropsychological assessment. That is, when selecting our assessment protocol, it is important to consider whether the task is capable of reproducing, at least partially, the natural conditions where spatial orientation occurs. Therefore, the tasks that seem closer to daily orientation activities are those that take place outdoors, in wide open spaces. However, the intrinsic characteristics of these tasks make them difficult to use. They have to be conducted in a specific large and natural space. Therefore, to conduct these tasks, it is 
necessary to interrupt the usual neuropsychological assessment, which normally takes place in a clinical setting. In addition, they are difficult to standardize and replicate, making their application difficult in a standard neuropsychological evaluation. Tasks that take place in a smaller room or enclosure lose functionality but gain in standardized application. However, it is necessary to consider that some of these tasks have the disadvantage of requiring some type of equipment or specific environmental modification (large curtains or panels in most cases). Finally, tasks based on new technologies are the simplest to apply and include in routine neuropsychological assessments, but they probably have less relationship with day-to-day spatial orientation and, therefore, are the least functional. Tasks that use new technologies but achieve greater functional validity due to mobility or other characteristics they provide to participants have the disadvantage of requiring specific and expensive apparatus, such as augmented reality or virtual reality equipment.

\section{Limitations}

It is necessary to point out the lack of consensus in the terminology used in spatial orientation tasks: we found terms such as self-reference, idiothetic, response learning, and so forth, referring to the use of the egocentric spatial orientation, and terms such as Euclidean properties, allothetic, place learning, and so forth, referring to the use of the allocentric spatial orientation. Even the concept of "spatial orientation" is often referred to in terms of "spatial memory," "reorientation," or "spatial navigation." This divergence in the terms used can make it difficult to obtain relevant bibliographical references. Moreover, it is important to mention that the main aim of some of the articles analyzed in the present review was not to assess spatial orientation per se, but rather, for example, the ability to use a map to transfer $2 \mathrm{D}$ information to 3D (Vasilyeva \& Bowers, 2006) or assess the topographical span (Piccardi, Leonzi, et al., 2014; Piccardi, Palermo, et al., 2014). In addition, other studies have shown interest in verifying or refuting geometry module processing (Gouteux \& Spelke, 2001; Gouteux et al., 2001; Hermer-Vazquez et al., 2001; Hupbach \& Nadel, 2005; Huttenlocher \& Vasilyeva, 2003; Learmonth et al., 2002, 2001, 2008; Lee \& Spelke, 2010, 2011; Lee, Winkler-Rhoades, et al., 2012; Lew et al., 2006; Lourenco et al., 2009; Lourenco \& Cabrera, 2015; Nardini, Atkinson, et al., 2008; Nardini et al., 2006; Newcombe et al., 2010;
Twyman et al., 2007; Vasilyeva \& Bowers, 2006; Yousif \& Lourenco, 2017), an innate ability, shared with other vertebrate animals, that employs geometrical features in encapsulated environments to successfully maintain correct orientation in space (Cheng \& Newcombe, 2005). The way geometry is used throughout the development of spatial orientation is undoubtedly a subject of vital importance, but it is probably a topic too specific to focus on in a neuropsychological evaluation and its subsequent diagnosis. Despite this, all these studies include tasks that require spatial orientation abilities, and for this reason they have been included in the present review.

Another possible limitation that we currently find in spatial orientation researches in childhood is the difficulty in establishing the development of egocentric and allocentric frames of reference. That is, that the authors choose a certain age range does not mean that the measured ability has not emerged before; for example, that authors assess a function after 5 years, but this is actually already present in children of 4 years. Thus, it could happen that the course of development of the spatial orientation shown in the present study does not correspond exactly with the ages when these capacities emerge and improve, but at lower ages that have not been measured in the original studies. For this reason, it could be recommendable in future researches to try to expand these evaluation tasks for younger ages.

We also need to highlight that the classification followed in the present review presents certain controversies. First, some authors do not clearly mention the frames of reference used in the solution of their experimental tasks, making it difficult to classify them in egocentric and/or allocentric terms. Regarding cue use, the differentiation between coincident cues (coding of the distinctive characteristics of the location) and noncoincident cues (coding of the target location in terms of its relationship with other reference points) seems clear, whereas the difference between proximal and distal cues is more diffuse. In spatial memory paradigms in rodents, taking into account the relative size of the animal vs. the size of the elements for the task-labyrinths and cues-it seems quite clear that the distal landmarks are those that are located outside the labyrinth, that is, those that do not vary their relative position while the organism moves (O'Keefe et al., 1978). This does not occur with spatial orientation in humans. As we have seen previously, many of the tasks that employ cues classified as distal are performed in closed rooms and only a few in open spaces. Therefore, it seems necessary to 
reconsider the extent to which we can talk about a distal cue when it is located a few meters away from the participant.

\section{Conclusion}

The purpose of the present review was to assess and summarize recent literature on egocentric and allocentric spatial orientation assessment in children less than 6 years of age. Results revealed a wide variety of tasks for the evaluation of spatial orientation at these early ages.

We can conclude that spatial orientation starts early in development, with the egocentric framework being the first to appear, as well as allocentric orientation using coincident landmarks. Infants of 4.5 months old are able to orient themselves with coincident proximal landmarks if they previously had the chance to know the environment from different points of view. Later, infants of 8.5 months old use noncoincident landmarks for the very first time, and these cues continue to guide the toddler's orientation from 12 to 18 months, if they have not been disoriented, and from 18 to 24 months if they have changed their position, starting to use geometrical and nongeometrical cues as well. From 2 to 3 years of age, although children continue to use different kinds of noncoincident landmarks, they show the use of distal cues to reorient themselves. From 3 to 4 years old, children's reorientation improves with the use of different proximal and distal noncoincident landmarks. Later, from 4 to 5 years old, they are able to compensate for the lack of self-motion in enclosed spaces or establish a spatial relationship between discontinued elements, and it seems that they start to integrate different types of spatial cues. From 5 years old onward, they do not show any problems with geometric, nongeometric, distal or proximal cues, they improve their ability to integrate difference sources of spatial information and they are able to remember more spatial information in their memory.

We have observed, despite this wide variety of tests, a lack of tasks to analyze all the relevant characteristics of daily spatial orientation in preschool ages. That is, whereas we have many tasks for allocentric evaluation, with different types of cues and aimed at different ages, we find that the current spatial orientation tasks for preschoolers fail to evaluate the egocentric framework, which highlights the need to develop tasks for the evaluation of the egocentric spatial orientation at early ages. Although in our daily activity, spatial orientation depends on the combination and use of both frameworks, it would be of great interest to have tasks available to evaluate egocentric and allocentric orientation separately. These tasks can detect developmental alterations in the management of a specific framework that could occur in neurological diseases or developmental problems that affect the main brain areas that participate in spatial orientation, such as hippocampal areas, temporal, parietal and temporal cortex, thalamus, and striatum. Some other relevant issues in spatial orientation assessment, such as whether children are allowed to move during the tasks or whether spatial tasks are carried out in real environments, are present in the most of spatial orientation test. Moreover, the incipient presence of new technologies in the evaluation of spatial orientation is not negligible. Therefore, it would be advisable to continue researching in this direction with the aim of ensuring an appropriate ecological validity of these procedures.

\section{Acknowledgments}

We thank Cynthia DePoy for revising the English text of the manuscript.

\section{Disclosure statement}

The authors declare that they have no conflict of interest.

\section{References}

Aanes, S., Bjuland, K. J., Skranes, J., \& Løhaugen, G. C. C. (2015). Memory function and hippocampal volumes in preterm born very-low-birth-weight (VLBW) young adults. NeuroImage, 105, 76-83. doi:10.1016/j.neuroimage.2014.10.023

Acredolo, L. P. (1978). Development of spatial orientation in infancy. Developmental Psychology, 14(3), 224-234. doi:10.1037/0012-1649.14.3.224

Acredolo, L. P., Adams, A., \& Goodwyn, S. W. (1984). The role of self-produced movement and visual tracking in infant spatial orientation. Journal of Experimental Child Psychology, 38(2), 312-327. doi:10.1016/00220965(84)90128-0

Acredolo, L. P., \& Evans, D. (1980). Developmental changes in the effects of landmarks on infant spatial behavior. Developmental Psychology, 16(4), 312-318. doi:10.1037/ 0012-1649.16.4.312

Banta Lavenex, P., Lecci, S., Pretre, V., Brandner, C., Mazza, C., Pasquier, J., \& Lavenex, P. (2011). As the world turns: Short-term human spatial memory in egocentric and allocentric coordinates. Behavioural Brain Research, 219(1), 132-141. doi:10.1016/j.bbr.2010.12.035

Beauchamp, M. H., Thompson, D. K., Howard, K., Doyle, L. W., Egan, G. F., Inder, T. E., \& Anderson, P. J. (2008). Preterm infant hippocampal volumes correlate with later 
working memory deficits. Brain, 131(11), 2986-2994. doi: 10.1093/brain/awn227

Belmonti, V., Fiori, S., Guzzetta, A., Cioni, G., \& Berthoz, A. (2015). Cognitive strategies for locomotor navigation in normal development and cerebral palsy. Developmental Medicine and Child Neurology, 57(s2), 31-36. doi:10.1111/dmcn.12685

Bernardino, I., Mouga, S., Castelo-Branco, M., \& Van Asselen, M. (2013). Egocentric and allocentric spatial representations in Williams Syndrome. Journal of the International Neuropsychological Society, 19(1), 54-62. doi:10.1017/S1355617712000963

Bianchini, F., Palermo, L., Piccardi, L., Incoccia, C., Nemmi, F., Sabatini, U., \& Guariglia, C. (2014). Where Am I? A new case of developmental topographical disorientation. Journal of Neuropsychology, 8(1), 107-124. doi:10.1111/ jnp. 12007

Bocchi, A., Palermo, L., Boccia, M., Palmiero, M., D’Amico, S., \& Piccardi, L. (2018). Object recognition and location: Which component of object location memory for landmarks is affected by gender? Evidence from four to ten year-old children. Applied Neuropsychology: Child, o(0), 1-10. doi:10.1080/21622965.2018.1504218

Boccia, M., Nemmi, F., \& Guariglia, C. (2014). Neuropsychology of environmental navigation in humans: Review and meta-analysis of fMRI studies in healthy participants. Neuropsychology Review, 24(2), 236-251. doi:10.1007/s11065-014-9247-8

*Bremner, J. G., Hatton, F., Foster, K. A., \& Mason, U. (2011). The contribution of visual and vestibular information to spatial orientation by 6- to 14-month-old infants and adults. Developmental Science, 14(5), 1033-1045. doi:10.1111/j.1467-7687.2011.01051.x

Broadbent, H. J., Farran, E. K., \& Tolmie, A. (2014). Egocentric and allocentric navigation strategies in Williams syndrome and typical development. Developmental Science, 17(6), 920-934. doi:10.1111/desc. 12176

Bull, R., Espy, K. A., \& Wiebe, S. A. (2008). Short-term memory, working memory, and executive functioning in preschoolers: Longitudinal predictors of mathematical achievement at age 7 years. Developmental Neuropsychology, 33(3), 205-228. doi:10.1080/ 87565640801982312

*Bullens, J., Iglói, K., Berthoz, A., Postma, A., \& RondiReig, L. (2010). Developmental time course of the acquisition of sequential egocentric and allocentric navigation strategies. Journal of Experimental Child, 107(3), 337-350. doi:10.1016/j.jecp.2010.05.010

*Bullens, J., Klugkist, I., \& Postma, A. (2011). The role of local and distal landmarks in the development of object location memory. Developmental Psychology, 47(6), 1515-1524. doi:10.1037/a0025273

*Bullens, J., Nardini, M., Doeller, C. F., Braddick, O., Postma, A., \& Burgess, N. (2010). The role of landmarks and boundaries in the development of spatial memory. Developmental Science, 13(1), 170-180. doi:10.1111/j. 1467-7687.2009.00870.x

Burgess, N. (2008). Spatial cognition and the brain. Annals of the New York Academy of Sciences, 1124(1), 77-97. doi:10.1196/annals.1440.002
Burgess, N., Maguire, E. A., \& O'Keefe, J. (2002). The human hippocampus and spatial and episodic memory. Neuron, 35(4), 625-641. doi:10.1016/S0896-6273(02) 00830-9

Caravale, B., Tozzi, C., Albino, G., \& Vicari, S. (2005). Cognitive development in low risk preterm infants at 3-4 years of life. Archives of Disease in Childhood - Fetal and Neonatal Edition, 90(6), 474-479. doi:10.1136/adc.2004. 070284

Chen, Y., Monaco, S., Byrne, P., Yan, X., Henriques, D. Y. P., \& Crawford, J. D. (2014). Allocentric versus egocentric representation of remembered reach targets in human cortex. Journal of Neuroscience, 34(37), 12515-12526. doi:10.1523/JNEUROSCI.1445-14.2014

Cheng, K., \& Newcombe, N. S. (2005). Is there a geometric module for spatial orientation? Squaring theory and evidence. Psychonomic Bulletin \& Review, 12(1), 1-23. doi: 10.3758/BF03196346

Cimadevilla, J. M., Roldán, L., París, M., Arnedo, M., \& Roldán, S. (2014). Spatial learning in a virtual realitybased task is altered in very preterm children. Journal of Clinical and Experimental Neuropsychology, 36(9), 1002-1008. doi:10.1080/13803395.2014.963520

Clohessy, A. B., Posner, M. I., \& Rothbart, M. K. (2001). Development of the functional visual field. Acta Psychologica, 106(1-2), 51-68. doi:10.1016/S00016918(00)00026-3

Committeri, G., Paradis, A. L., Galati, G., Berthoz, A., LeBihan, D., \& Pizzamiglio, L. (2004). Reference frames for spatial cognition: Different brain areas are involved in viewer-, object-, and landmark-centered judgments about object location. Journal of Cognitive Neuroscience, 16(9), 1517-1535. doi:10.1162/0898929042568550

Conson, M., Bianchini, F., Quarantelli, M., Salzano, S., Di, A., \& Guariglia, C. (2018). Selective map-following navigation deficit: A new case of developmental topographical disorientation. Journal of Clinical and Experimental Neuropsychology, 40, 940-950. doi:10.1080/13803395.2018. 1451493

*Crowther, H. L., Lew, A. R., \& Whitaker, C. J. (2000). The development of beacon use for spatial orientation in 6 8. 5-month-old infants. Infant Behavior and Development, 23(1), 41-59.

Demir, Ö. E., Prado, J., \& Booth, J. R. (2014). The differential role of verbal and spatial working memory in the neural basis of arithmetic. Developmental Neuropsychology, 39(6), 440-458. doi:10.1080/87565641. 2014.939182

Diamond, A., \& Goldman-Rakic, P. S. (1989). Comparison of human infants and rhesus monkeys on Piaget's $A B$ task: Evidence for dependence on dorsolateral prefrontal cortex. Experimental Brain Research, 74(1), 24-40. doi:10.1007/BF00248277

Doeller, C. F., King, J. A., \& Burgess, N. (2008). Parallel striatal and hippocampal systems for landmarks and boundaries in spatial memory. Proceedings of the National Academy of Sciences, 105(15), 5915-5920. doi: 10.1073/pnas.0801489105

Garon, N., Bryson, S. E., \& Smith, I. M. (2008). Executive function in preschoolers: A review using an integrative framework. Psychological Bulletin, 134(1), 31-60. doi:10.1037/0033-2909.134.1.31 
Gilmore, R., \& Johnson, M. H. (1995). Working memory in infancy 6 month-olds performance on two versions of the oculomotor delayed response task. Journal of Experimental Child Psychology, 59, 397-418.

*Gouteux, S., \& Spelke, E. S. (2001). Children's use of geometry and landmarks to reorient in an open space. Cognition, 81(2), 119-148. doi:10.1016/S0010-0277(01) 00128-7

*Gouteux, S., Vauclair, J., \& Thinus-Blanc, C. (2001). Reorientation in a small-scale environment by 3-4-, and 5-year-old children. Cognitive Development, 16(3), 853-869. doi:10.1016/s0885-2014(01)00062-4

Gramann, K., Onton, J., Riccobon, D., Mueller, H. J., Bardins, S., \& Makeig, S. (2010). Human brain dynamics accompanying use of egocentric and allocentric reference frames during navigation. Journal of Cognitive Neuroscience, 22(12), 2836-2849. doi:10.1162/jocn.2009. 21369

Hamilton, D. A., Kodituwakku, P., Sutherland, R. J., \& Savage, D. D. (2003). Children with Fetal Alcohol Syndrome are impaired at place learning but not cuednavigation in a virtual Morris water task. Behavioural Brain Research, 143(1), 85-94. doi:10.1016/S01664328(03)00028-7

*Hermer-Vazquez, L., Moffet, A., \& Munkholm, P. (2001). Language, space, and the development of cognitive flexibility in humans: The case of two spatial memory tasks. Cognition, 79(3), 263-299. doi:10.1016/S00100277(00)00120-7

*Hupbach, A., \& Nadel, L. (2005). Reorientation in a rhombic environment: No evidence for an encapsulated geometric module. Cognitive Development, 20(2), 279-302. doi:10.1016/j.cogdev.2005.04.003

*Huttenlocher, J., \& Vasilyeva, M. (2003). How toddlers represent enclosed spaces. Cognitive Science, 27(5), 749-766. doi:10.1016/S0364-0213(03)00062-4

Iaria, G., \& Barton, J. J. S. (2010). Developmental topographical disorientation: A newly discovered cognitive disorder. Experimental Brain Research, 206(2), 189-196. doi:10.1007/s00221-010-2256-9

Iaria, G., Bogod, N., Fox, C. J., \& Barton, J. J. S. (2009). Neuropsychologia Developmental topographical disorientation: Case one. Neuropsychologia, 47(1), 30-40. doi:10.1016/j.neuropsychologia.2008.08.021

Iaria, G., Incoccia, C., Piccardi, L., Nico, D., Sabatini, U., \& Guariglia, C. (2005). Lack of orientation due to a congenital brain malformation: A case study. Neurocase, 11(6), 463-474. doi:10.1080/13554790500423602

*Juan, M. C., Mendez-Lopez, M., Perez-Hernandez, E., \& Albiol-Perez, S. (2014). Augmented reality for the assessment of children's spatial memory in real settings. PLoS One, 9(12), 1-26. doi:10.1371/journal.pone.0113751

*Kaufman, J., \& Needham, A. (2011). Spatial expectations of young human infants, following passive movement. Developmental Psychobiology, 53(1), 23-36. doi:10.1002/ dev.20484

Lambert, F. R., Lavenex, P., \& Banta Lavenex, P. (2015). Improvement of allocentric spatial memory resolution in children from 2 to 4 years of age. International Journal of Behavioral Development, 39(4), 318-331. doi:10.1177/ 0165025415584808
Lavenex, P. B., Bostelmann, M., Brandner, C., Costanzo, F., Fragnière, E., Klencklen, G., ... Vicari, S. (2015). Allocentric spatial learning and memory deficits in Down syndrome. Frontiers in Psychology, 6(FEB), 1-17. doi:10.3389/fpsyg.2015.00062

*Learmonth, A. E., Nadel, L., \& Newcombe, N. S. (2002). Children's use of landmarks: Implication for modularity theory. Psychological Science, 13(4), 337-341.

Learmonth, A. E., Newcombe, N. S., \& Huttenlocher, J. (2001). Toddlers' use of metric information and landmarks to reorient. Journal of Experimental Child Psychology, 80(3), 225-244. doi:10.1006/jecp.2001.2635

Learmonth, A. E., Newcombe, N. S., Sheridan, N., \& Jones, M. (2008). Why size counts: Children's spatial reorientation in large and small enclosures. Developmental Science, 11(3), 414-426. doi:10.1111/j.1467-7687.2008.00686.x

*Lee, S. A., Sovrano, V. A., \& Spelke, E. S. (2012). Navigation as a source of geometric knowledge: Young children's use of length, angle, distance, and direction in a reorientation task. Cognition, 123(1), 144-161. doi:10.1016/j.cognition.2011.12.015

${ }^{*}$ Lee, S. A., \& Spelke, E. S. (2010). A modular geometric mechanism for reorientation in children. Cognitive Psychology, 61(2), 152-176. doi:10.1016/j.cogpsych.2010. 04.002

${ }^{*}$ Lee, S. A., \& Spelke, E. S. (2011). Young children reorient by computing layout geometry, not by matching images of the environment. Psychonomic Bulletin of Review, 18(1), 192-198. doi:10.3758/s13423-010-0035-Z

*Lee, S. A., Winkler-Rhoades, N., \& Spelke, E. S. (2012). Spontaneous reorientation is guided by perceived surface distance, not by image matching or comparison. PLoS One, 7(12), 1-8. doi:10.1371/journal.pone.0051373

*León, I., Cimadevilla, J. M., \& Tascón, L. (2014). Developmental gender differences in children in a virtual spatial memory task. Neuropsychology, 28(4), 485-495. doi:10.1037/neu0000054

*Leplow, B., Lehnung, M., Pohl, J., Herzog, A., Ferstl, R., \& Mehdorn, M. (2003). Navigational place learning in children and young adults as assessed with a standardized locomotor search task. British Journal of Psychology, 94(3), 299-317. doi:10.1348/000712603767876244

*Lew, A. R., Bremner, J. G., \& Lefkovitch, L. P. (2000). The development of relational landmark use in six- to twelvemonth-old infants in a spatial orientation task. Child Development, 71(5), 1179-1190. doi:10.1111/1467-8624. 00222

*Lew, A. R., Foster, K. A., \& Bremner, J. G. (2006). Disorientation inhibits landmark use in 12-18-month-old infants. Infant Behavior and Development, 29(3), 334-341. doi:10.1016/j.infbeh.2006.01.001

*Lourenco, S. F., Addy, D., \& Huttenlocher, J. (2009). Location representation in enclosed spaces: What types of information afford young children an advantage? Journal of Experimental Child Psychology, 104(3), 313-325. doi: 10.1016/j.jecp.2009.05.007

*Lourenco, S. F., \& Cabrera, J. (2015). The potentiation of geometry by features in human children: Evidence against modularity in the domain of navigation. Journal of Experimental Child Psychology, 140, 184-196. doi:10.1016/j.jecp.2015.07.007
2066

2067

2068

2069

2070

2071

2072

2073

2074

2075

2076

2077

2078

2079

2080

2081

2082

2083

2084

2085

2086

2087

2088

2089

2090

2091

2092

2093

2094

2095

2096

2097

2098

2099

2100

2101

2102

2103

2104

2105

2106

2107

2108

2109

2110

2111

2112

2113

2114

2115

2116

2117

2118 
*Mandolesi, L., Petrosini, L., Menghini, D., Addona, F., \& Vicari, S. (2009). Children's radial arm maze performance as a function of age and sex. International Journal of Developmental Neuroscience, 27(8), 789-797. doi:10.1016/j.ijdevneu.2009.08.010

*Merrill, E. C., Yang, Y., Roskos, B., Steele, S., \& Farran, E. K. (2016). Sex differences in using spatial and verbal abilities influence route learning performance in a virtual environment: A comparison of 6- to 12-year old boys and girls. Frontiers in Psychology, 7(February), 1-17. doi:10.3389/fpsyg.2016.00258

Montello, D. R. (2005). Navigation. In P. Shah \& A. Miyake (Eds.), Handbook of visuospatial thinking (pp. 257-294). Cambridge, MA: Cambrigde University Press.

Moulinet, A. R., Chochina, L., Leplaideur, S. C., Bannier, E., Ferre, J.-C., \& Bonan, I. (2016). Cortical activity and egocentric representation: An fMRI study. Annals of Physical and Rehabilitation Medicine, 59, e70. doi:10.1016/j.rehab. 2016.07.162

Mürner-Lavanchy, I., Ritter, B. C., Spencer-Smith, M. M., Perrig, W. J., Schroth, G., Steinlin, M., \& Everts, R. (2014). Visuospatial working memory in very preterm and term born children-Impact of age and performance. Developmental Cognitive Neuroscience, 9, 106-116. doi:10.1016/j.den.2014.02.004

*Nardini, M., Atkinson, J., \& Burgess, N. (2008). Children reorient using the left/right sense of coloured landmarks at 18-24 months. Cognition, 106(1), 519-527. doi:10.1016/ j.cognition.2007.02.007

*Nardini, M., Burgess, N., Breckenridge, K., \& Atkinson, J. (2006). Differential developmental trajectories for egocentric, environmental and intrinsic frames of reference in spatial memory. Cognition, 101(1), 153-172. doi:10.1016/ j.cognition.2005.09.005

*Nardini, M., Jones, P., Bedford, R., \& Braddick, O. (2008). Development of cue integration in human navigation. Current Biology, 18(9), 689-693. doi:10.1016/j.cub.2008. 04.021

*Negen, J., Heywood-Everett, E., Roome, H. E., \& Nardini, M. (2018). Development of allocentric spatial recall from new viewpoints in virtual reality. Developmental Science, 21(1), 1-12. doi:10.1111/desc.12496

Neggers, S. F. W., Van der Lubbe, R. H. J., Ramsey, N. F., \& Postma, A. (2006). Interactions between ego- and allocentric neuronal representations of space. NeuroImage, 31(1), 320-331. doi:10.1016/j.neuroimage.2005.12.028

Nemmi, F., Bianchini, F., Piras, F., Péran, P., Palermo, L., Piccardi, L., ... Guariglia, C. (2015). Finding my own way: An fMRI single case study of a subject with developmental topographical disorientation. Neurocase, 21(5), 573-583. doi:10.1080/13554794.2014.960424

Nemmi, F., Boccia, M., \& Guariglia, C. (2017). Does aging affect the formation of new topographical memories? Evidence from an extensive spatial training. Neuropsychology, Development, and Cognition. Section B, Aging, Neuropsychology and Cognition, 24(1), 29-44. doi: 10.1080/13825585.2016.1167162

*Newcombe, N., Huttenlocher, J., Drummey, A. B., \& Wiley, J. G. (1998). The development of spatial location coding: Place learning and dead reckoning in the second and third years. Cognitive Development, 13(2), 185-200. doi:10.1016/S0885-2014(98)90038-7
Newcombe, N. S., Ratliff, K. R., Shallcross, W. L., \& Twyman, A. D. (2010). Young children's use of features to reorient is more than just associative: Further evidence against a modular view of spatial processing. Developmental Science, 13(1), 213-220. doi:10.1111/j. 1467-7687.2009.00877.x

Nunes, M. M., Honjo, R. S., Dutra, R. L., Amaral, V. S., Amaral, V. A. S., Oh, H. K., ... Teixeira, M. C. T. V. (2013). Assessment of intellectual and visuo-spatial abilities in children and adults with Williams syndrome. Universitas Psychologica, 12(2), 581-589. doi:10.11144/ Javeriana.UPSY12-2.aiva

O'Keefe, J. Nadel., \& Lynn, (1978). The hippocampus as a cognitive map. Oxford, UK: Clarendon Press.

Oakes, L. M., Hurley, K. B., Ross-Sheehy, S., \& Luck, S. J. (2011). Developmental changes in infants' visual shortterm memory for location. Cognition, 118(3), 293-305. doi:10.1016/j.cognition.2010.11.007.Developmental

Overman, W. H., Pate, B. J., Moore, K., \& Peuster, A. (1996). Ontogeny of place learning in children as measured in the radial arm maze. Behavioral Neuroscience, 110(6), 1205-1228. doi:10.1037/0735-7044.110.6.1205

Palermo, L., Foti, F., Ferlazzo, F., Guariglia, C., \& Petrosini, L. (2014). I find my way in a maze but not in my own territory! Navigational processing in developmental topographical disorientation. Neuropsychology, 28(1), 135-146. doi:10.1037/neu0000021

Palermo, L., Piccardi, L., Bianchini, F., Nemmi, F., Incoccia, C., Sabatini, U., \& Guariglia, C. (2014). Looking for the compass in a case of developmental topographical disorientation: A behavioral and neuroimaging study. Journal of Clinical and Experimental Neuropsychology, 36(5):464-481. doi:10.1080/13803395.2014.904843

Pelphrey, K. A., Reznick, J. S., Goldman, B. D., Sasson, N., Morrow, J., Donahoe, A., \& Hodgson, K. (2004). Development of visuospatial short-term memory in the second half of the 1st year. Developmental Psychology, 40(5), 836-851. doi:10.1037/0012-1649.40.5.836

*Pentland, L. M., Anderson, V. A., Dye, S., \& Wood, S. J. (2003). The Nine Box Maze Test: A measure of spatial memory development in children. Brain and Cognition, 52(2), 144-154. doi:10.1016/S0278-2626(03)00079-4

Piaget, J., \& Inhelder, B. (1967). A child's conception of space (L. Angdon \&F. J. Lunzer, Eds.). New York, NY: Norton.

Piccardi, L., Iaria, G., Ricci, M., Bianchini, F., Zompanti, L., \& Guariglia, C. (2008). Walking in the Corsi test: Which type of memory do you need? Neuroscience Letters, 432(2), 127-131. doi:10.1016/j.neulet.2007.12.044

*Piccardi, L., Leonzi, M., D’Amico, S., Marano, A., \& Guariglia, C. (2014). Development of navigational working memory: Evidence from 6- to 10-year-old children. British Journal of Developmental Psychology, 32(2), 205-217. doi:10.1111/bjdp.12036

*Piccardi, L., Palermo, L., Bocchi, A., \& Guariglia, C. (2015). Does spatial locative comprehension predict landmark-based navigation? PLoS One. 10(1), e0115432. doi: 10.1371/journal.pone.0115432

Piccardi, L., Palermo, L., Leonzi, M., Risetti, M., Zompanti, L., D'Amico, S., \& Guariglia, C. (2014). The walking Corsi test (WalCT): A normative study of topographical working memory in a sample of 4-to 11-Year-Olds.
2172

2173

2174

2175

2176

2177

2178

2179

2180

2181

2182

2183

2184

2185

2186

2187

2188

2189

2190

2191

2192

2193

2194

2195

2196

2197

2198

2199

2200

2201

2202

2203

2204

2205

2206

2207

2208

2209

2210

2211

2212

2213

2214

2215

2216

2217

2218

2219

2220

2221

2222

2223

2224 
Clinical Neuropsychologist, 28(1), 84-96. doi:10.1080/ 13854046.2013.863976

Reynolds, G. D., \& Romano, A. C. (2016). The development of attention systems and working memory in infancy. Frontiers in Systems Neuroscience, 10(March), 1-12. doi: 10.3389/fnsys.2016.00015

Reznick, J. S., Morrow, J. D., Goldman, B. D., \& Snyder, J. (2004). The onset of working memory in infants. Infancy, 6(1), 145-154. doi:10.1207/s15327078in0601_7

*Ribordy, F., Jabès, A., Banta Lavenex, P., \& Lavenex, P. (2013). Development of allocentric spatial memory abilities in children from 18 months to 5 years of age. Cognitive Psychology, 66(1), 1-29. doi:10.1016/j.cogpsych. 2012.08.001

Ribordy Lambert, F., Lavenex, P., \& Banta Lavenex, P. (2017). The "when" and the "where" of single-trial allocentric spatial memory performance in young children: Insights into the development of episodic memory. Developmental Psychobiology, 59(2), 185-196. doi:10.1002/dev.21479

Rieser, J. J. (1979). Spatial orientation of six-month-old infants. Child Development, 50(4), 1078-1087. doi:10.2307/ 1129334

*Rodriguez-Andres, D., Juan, M. C., Mendez-Lopez, M., Perez-Hernandez, E., \& Lluch, J. (2016). MnemoCity task: Assessment of childrens spatial memory using stereoscopy and virtual environments. PLoS ONE, 11(8), 1-28. doi:10.1371/journal.pone.0161858

Rodriguez-Andres, D., Mendez-Lopez, M., Juan, M. C., \& Perez-Hernandez, E. (2018). A virtual object-location task for children: Gender and videogame experience influence navigation; age impacts memory and completion time. Frontiers in Psychology, 9(APR), 1-13. doi:10.3389/fpsyg. 2018.00451

Ruby, P., \& Decety, J. (2001). Effect of subjective perspective taking during simulation of action: A PET investigation of agency. Nature Neuroscience, 4(5), 1-5.

*Ruggiero, G., D'Errico, O., \& Iachini, T. (2016). Development of egocentric and allocentric spatial representations from childhood to elderly age. Psychological Research, 80(2), 259-272. doi:10.1007/s00426-015-0658-9

Saj, A., Cojan, Y., Musel, B., Honoré, J., Borel, L., \& Vuilleumier, P. (2014). Functional neuro-anatomy of egocentric versus allocentric space representation. Neurophysiologie Clinique, 44(1), 33-40. doi:10.1016/j. neucli.2013.10.135

Schmuckler, M. A., \& Jewell, S. (2007). The effect of simulated self versus object movement in a nonsearch task. Infancy, 11(3), 305-320. doi:10.1111/j.1532-7078.2007. tb00229.x

*Schmuckler, M. A., \& Tsang-Tong, H. Y. (2000). The role of visual and body movement information in infant search. Developmental Psychology, 36(4), 499-510. doi:10. 1037/0012-1649.36.4.499

Siegel, A. W., \& White, S. H. (1975). The development of spatial representations of large-scale environments. In H. W. Reese (Ed.), Advances in child development \& behavior (pp. 9-55). New York, NY: Academy Press.

*Smith, A. D., Gilchrist, I. D., Cater, K., Ikram, N., Nott, K., \& Hood, B. M. (2008). Reorientation in the real world: The development of landmark use and integration in a natural environment. Cognition, 107(3), 1102-1111. doi:10.1016/j.cognition.2007.10.008

Tascón, L., Castillo, J., León, I., \& Cimadevilla, J. M. (2018). Walking and non-walking space in an equivalent virtual reality task: Sexual dimorphism and aging decline of spatial abilities. Behavioural Brain Research, 347, 201-208. doi:10.1016/j.bbr.2018.03.022

*Twyman, A., Friedman, A., \& Spetch, M. L. (2007). Penetrating the geometric module: Catalyzing children's use of landmarks. Developmental Psychology, 43(6), 1523-1530. doi:10.1037/0012-1649.43.6.1523

*van den Brink, D., \& Janzen, G. (2013). Visual spatial cue use for guiding orientation in two-to-three-year-old children. Frontiers in Psychology, 4, 1-12. doi:10.3389/fpsyg. 2013.00904

*Vasilyeva, M., \& Bowers, E. (2006). Children's use of geometric information in mapping tasks. Journal of Experimental Child Psychology, 95(4), 255-277. doi:10.1016/ j.jecp.2006.05.001

Vasilyeva, M., \& Lourenco, S. F. (2012). Development of spatial cognition. Wiley Interdisciplinary Reviews: Cognitive Science, 3(3), 349-362. doi:10.1002/wcs.1171

Vicari, S., Bellucci, S., \& Carlesimo, G. A. (2003). Visual and spatial working memory dissociation: Evidence from Williams syndrome. Developmental Medicine and Child Neurology, 45(4), 269-273. doi:10.1017/S001216220 3000513

*Waismeyer, A. S., \& Jacobs, L. F. (2013). The emergence of flexible spatial strategies in young children. Developmental Psychology, 49(2), 232-242. doi:10.1037/ a0028334

*Yang, Y., Merrill, E. C., \& Wang, Q. (2019). Children's response, landmark, and metric strategies in spatial navigation. Journal of Experimental Child Psychology, 181, 75-101. doi:10.1016/j.jecp.2019.01.005

*Yousif, S. R., \& Lourenco, S. F. (2017). Are all geometric cues created equal? Children's use of distance and length for reorientation. Cognitive Development, 43, 159-169. doi:10.1016/j.cogdev.2017.04.001

Zaehle, T., Jordan, K., Wüstenberg, T., Baudewig, J., Dechent, P., \& Mast, F. W. (2007). The neural basis of the egocentric and allocentric spatial frame of reference. Brain Research, 1137, 92-103. doi:10.1016/j.brainres.2006. 12.044
2278

2279

2280

2281

2282

2283

2284

2285

2286

2287

2288

2289

2290

Q3 2291

2292

2293

2294

2295

2296

2297

2298

2299

2300

2301

2302

2303

2304

2305

2306

2307

2308

2309

2310

2311

2312

2313

2314

2315

2316

2317

2318

2319

2320

2321

2322

2323

2324

2325

2326

2327

2328

2329

2330 